(Physiologisches Institut der Universität Bologna.)

\title{
Ueber die Nahrungs- und Stoffwechselbilanz des italienischen Bauers.
}

\author{
Untersuchungen von
}

\section{Peter Albertomi und Ivo Novi.}

Die gegenwärtigen experimentellen Untersuebungen bezwecken die Feststellung der Schwankungen der Nahrungsbilan\% unter natürlichen Bedingungen und die Bestimmung des für die Stickstoffbilanz ausreichenden Minimum, eine Frage von sozialem Interesse. Die Schaffung eines einzigen Menschentypus, dessen Bilanz verschieden sein soll, je nachdem das Individuum arbeitet oder ruht, gesund oder reconvalescent ist, ist nicht natürlich, weil die Nahrungsbilanz von Individuum zu Individum mit der Beschaffenheit und Zubereitung der Nahrungsmittel, mit den Jahreszeiten und einer Unzahl von Nebenbedingungen sich ändert. Zar Schöpfung eines einzigen Typus sind Untersuchungen unter den verschiedendsten bekannten Verhältnissen erforderlich, und wenn man auch nach Berechnung der Durchschnittszahlen diesen Typus gebildet hätte, welcher die verschiedensten Einfliusse in sich vereinigen sollte, so wäre man antiphysiologisch verfahren, weil daraus ein Mensch resultiren würde, welcher unmöglich existiren kann. Wir ziehen es vor, für sich die Bilanzen bestimmter Menschenklassen unter bestimmten Bedingungen, wie sie die Aussenwelt und die Arbeit schaffen, zu erforschen. Hauptsächlich der armen Landbevölkerung, welche durch die Noth gezwangen ist, die strengste Gleichförmigkeit in den Lebensgewohnheiten zu befolgen, wandten wir unser Augenmerk zu, in der festen Zuversicht, auf diese Weise über die minimale mögliche Stickstoffbilanz Aufschluss zu erhalten. Man kann behaupten, dass zu der Zeit, wo unsere Abhandlung begonnen wurde, diesbezügliche nach neuen Methoden angestellte Untersuchungen mangelten.

In einem dem Kriegsminister vorgelegten Referate (1883) er* E. Pflüger, Archiv f. Physiologie. Ba. 56 . 
örtert J. Moles chott ${ }^{1}$ ) die Frage der Ration des italienischen Soldaten. Seine Angaben haben einen beschränkten Werth, weil die in den täglichen Rationen enthaltenen Nährstoffe nicht analytiseh bestimmt, sondern nach der $\mathrm{K}$ ö $\mathrm{n}$ i g'schen Tabelle berechnet wurden; ferner fehlen Angaben über die Assimilation von Seiten des Darmkanals und über den mit dem Harne ausgeschiedenen Stickstoff. Bekanntlich erwachsen daraus nicht unerbebliche Feblerquellen. Trotzdem führen wir Moleschott's Zahlen an:

Tägliche Ration des italienischen Soldaten (Linie) in Friedenszeit

Tägliche

\begin{tabular}{|c|c|c|c|c|c|}
\hline & Nahrungsmenge & Eiweissstoffe & Fett & Amylacea & Anorg. Subs \\
\hline Fleisch & gr 180 & 38,5 & 9,34 & - & 2,10 \\
\hline Reis & gr 150 & 7,6 & 1,13 & 125 & 0,75 \\
\hline \multirow[t]{3}{*}{ Brot } & gr 918 & 57,2 & 2,02 & 472 & 11,85 \\
\hline & & 103,3 & 12,49 & 597 & 14,70 \\
\hline & oder bei & \multicolumn{4}{|c|}{ Ersatz von Reis durch Mehlteig } \\
\hline Fleisch & gr 180 & 38,5 & 9,34 & - & 2,10 \\
\hline Mehlteig & gr 150 & 19,6 & 0,42 & 115 & 1,26 \\
\hline \multirow[t]{2}{*}{ Brot } & gr 918 & 57,2 & 2,02 & 472 & 11,85 \\
\hline & & 115,3 & 11,78 & 587 & 15,21 \\
\hline
\end{tabular}

Nach Mo le schott erhält der italienische Soldat im Vergleiche zur normalen Durchnittszahl:

$\begin{array}{lcc} & \text { Eiweissstofe } & \begin{array}{c}\text { Fett u. Amylacea } \\ \text { trocken berechnet }\end{array} \\ \text { bei mässiger Arbeit } & -12 & +23 \\ \text { bei mühevoller }, & -25 & -68 \\ \text { bei übermässiger , } & -40 & -306\end{array}$

Gerade während wir unsere Experimente anstellten, erschienen zwei wichtige Arbeiten über diesen Gegenstand aus der Schule von $\mathrm{De} G$ iaxa. Die erste ist von $\mathrm{De} G$ i a $\times \mathrm{a}^{2}$ ) selbst verfasst, welcher behufs Erforschung des Wesens der Pellagra in loco nach

1) Sulla razione del Soldato Italiano - Relazione al Ministro della Guerra - Rivista militare Italiana 1883.

2) De Giaxa, Contributo alle cognizioni sulla eziologia della Pellagra I Parte (Annali dell' Istituto d'Igiene di Roma). - Vol. II Nuova serie fasc. I 1893. 
den erforderlichen Massregeln die Nahrung der Banern in Ortschaften, welehe von Pellagra heimgesucht sind, wie Conegliano untersuchte. Die Nahrung bestand grösstentheils aus Polenta, Bohnen, Oel, Salz, Pfeffer und die Beobachtung dauerte drei Tage. Durchschnittlich wurden täglich eingeführt : Stickstoffsubstanzen gr. 117,6, Fett gr. 64,6, Kohlenhydrate 619,6, und die Analyse der Faeces bewies, dass die durchschnittliche tägliche Menge von Nährstoffen, welche assimilirt worden waren für die Stickstoffsubstanzen gr. 87,6, fuir das Fett gr 64,4, für die Kohlenhydrate gr 561,4 betrug. Diese Nahrung würde, wie D e G i a x a richtig bemerkt, als Product der physiologischen Verbrennung die Entwicklung von

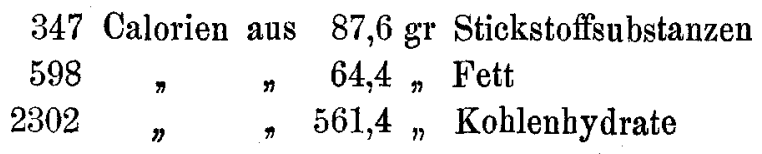

liefern, im Ganzen 3247 Calorien, eine Zahl, welche die von Voit für ein Individuum mit einem Körpergewichte von 70-75 Kilo berechnete (2810) übersteigt. Bei diesen Untersuchungen von D e G i a $\times$ a vermissen wir die der ausgeschiedenen Stickstoffmenge entsprechende Quantität. Wichtiger und erschöpfender ist die Arbeit von Manfredi ${ }^{1}$ ), welcher seine Versuche an Personen der niedersten Volksschichten in Neapel ausfuhrte, denen die Nahrung: vom Wirtshause oder von der Familie zubereitet wurde. Der für die Nahrung ausgegebene Betrag kommt uns ziemlich bedeutend vor, wenn man bedenkt, wie knapp die gewöhnlichen Einnahmen des Volkes in Italien bemessen sind; jede Person gab für die tägliche Nabrung 48, 60, 65, 75, 85 Centimes aus. Die tägliche Durchschnittsbilanz der acht untersuchten Individuen (zwei Schuster, eine alte Bettlerin, ein Tischler, eine Magd, ein Maurer, ein Lazzarone, eine wandernde Verkäuferin) war folgende:

1) Manfredi, Sulla nutrizione delle classi povere del po. polo in Napoli - Annali dell' Istituto di Igiene a Roma Vol. III Nuova serie fasc. I 1893. 


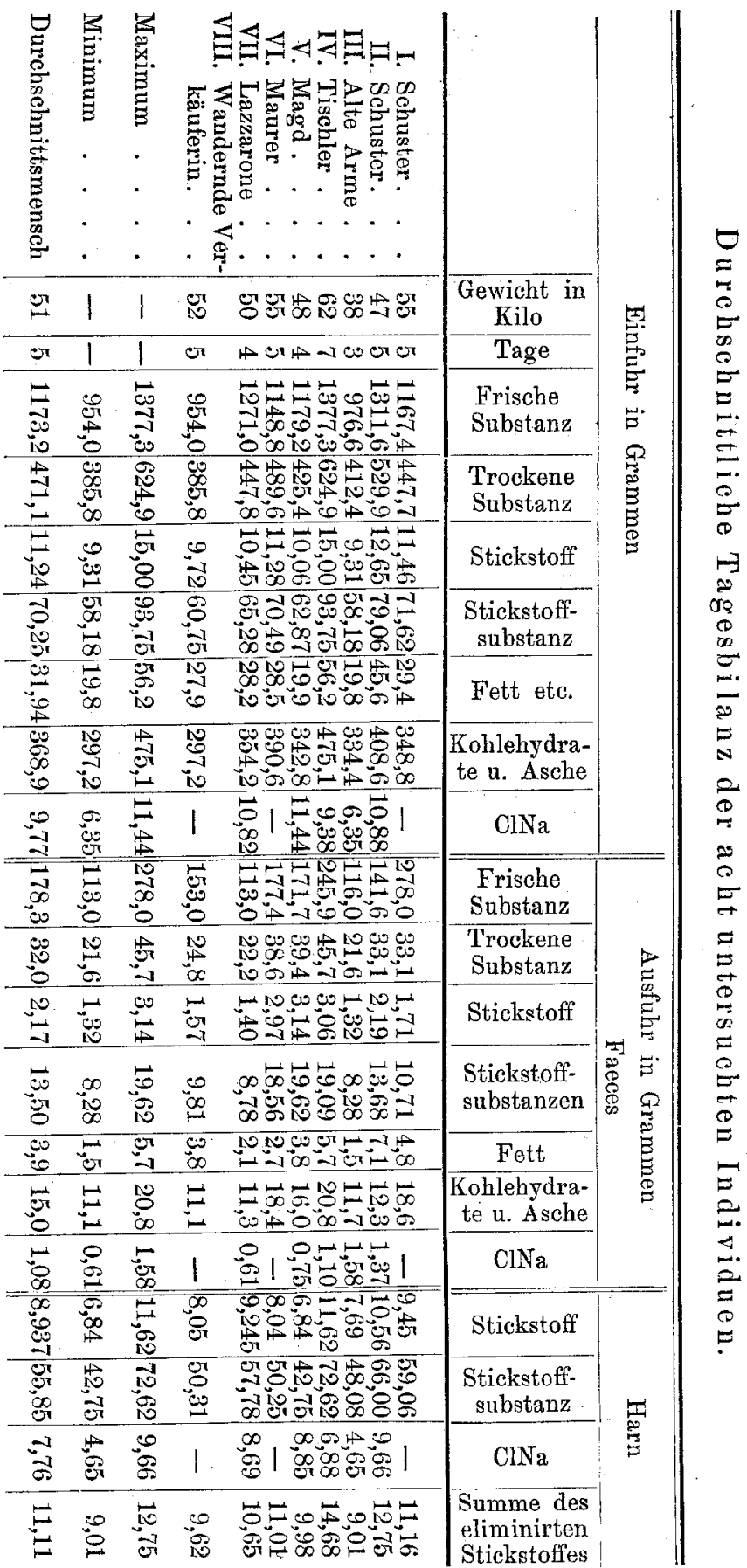


Eigene Versuche.

Unsere Experimente betreffen Feldarbeiter; welche in einer Sommer- und einer Winterperiode unter den von denselben gewöhnlich befolgten Lebensbedingungen beobachtet wurden. Vor Allem sorgten wir dafür, dass die gewöhnliche Nahrung der als Versuchsobjekte gewählten Bauern nicht im Geringsten geändert werde, und dass sie thatsächlich den Minima and Maxima der täglichen Einnahmen entspreche. Wir hielten es für angezeigt, sowohl die Sommer-, als auch die Winterbilanz zu ermitteln, weil in diesen zwei Jahreszeiten Arbeit und Ernährung sehr ver. schieden sind, daher auch Einnahmen und Ausgaben eine Aenderung erfahren müssen. Der italienische Bauer arbeitet und gewinnt im Winter weniger, isst etwas mehr als er gewinnt, verfällt somit in Schulden. Es ist selbstverständlich, dass er bestrebt sein wird, möglichst wenig zu essen, um nicht eine Schuld anzuhäufen, die er dann nicht tilgen kann. Im Sommer findet das Gegentheil statt, dem Bauer steht eine grössere Stundenzahl zur Verfügung, die Arbeit rentirt mehr und demnach arbeitet, isst, verbraucht er mehr und spart im Sommer.

\section{§ I. Nähere Angaben über die zum Experimente herbei- gezogenen Individuen.}

Die drei Personen, welche für unsere Versuche bestimmt waren, bilden eine Familie Feldarbeiter, sogenannter disobbligati (Tagelöhner), denen bisweilen im Winter Arbeit und Lohn abgehen. Im folgenden Prospekte führen wir den Lohn dieser Feldarbeiter an, wie erwachsene Männer, Weiber und Knaben uber dreizehn Jabre solchen bekommen.

Einnahmen des Mannes.

\begin{tabular}{lcccc} 
& Arbeitstage & Lohn & Totale Einnahme \\
October, November, Dezember & 74 & 0,70 & Fcs. 51,8 \\
Januar, Februar & 49 & 0,60 & $"$ & 28,8 \\
März, April & 48 & 0,70 & $"$ & 34,3 \\
Mai & 25 & 0,80 & $"$ & 20,0 \\
Juni & 23 & 1,00 & $"$ & 23,0 \\
Juli, August, September & 73 & 1,50 & & "109,5 \\
\hline
\end{tabular}

Fes. 267,4 
Berechnet man 30 Tage, wo die Arbeit unmöglìeh war, mit einem Durchsehnittslohne von $0,70 \mathrm{Fcs}$., so ergiebt sich als Nettoeinnahme des Mannes Fes. 267,4 - Fes. 21 = Fes. 246,40.

Einnahmen der Weiber und Knaben.

October, November

Januar, Februar, März

April, Mai

Juni

Juli

August, September
Arbeitstage

30

$\left.0^{1}\right)$

50

20

Lohn

Gesammtertrag

0,60

Fes. 18,0

0

0,60

0,60

, 0,0

$\Rightarrow \quad 30,0$

- Ueberbleibsel " 15,0

$"$

45,0

Fes. 120,0

Fes. 246,40

$" \quad, \quad$ Weibes

Summe
" 120,0

$" 120,0$

Fes. 486,40

Die ersten Versuche begannen am 3. März 1892. Das Familienbaupt, ein gewisser Carletti, ist ein gesunder, kräftiger 39jähriger Mann, welcher auf dem Lande in der Nähe von Ferrara ansässig ist, ist $168 \mathrm{~cm}$ hoch, wiegt im nüchternen Zustande $68,100 \mathrm{Kilo}$, ist rechtshändig und zeigt am Dynamometer eine Druckkraft' von 34 Kilogramm an. Die Körperoberfläche beträgt $1,932,3 \mathrm{~m}$. Seine Gemahlin ist 38 Jahre alt, erfrente sich immer ausgezeichneter Gesundheit und machte sechs Geburten durch, ist rechtsuändig und zeigt am Dynamometer eine Druckkraft von $36 \mathrm{Kilo}$ an; ist $152 \mathrm{~cm}$ hoch, wiegt 50,600 Kilo im nüchternen $\mathrm{Zu}$ stande. Die Hauptoberfläche beträgt 1,0668 m. Der Knabe, Sohn dieses Ehepaares, ist 14 Jahre alt, wiegt $34,800 \mathrm{Kilo}$, ist $140 \mathrm{~cm}$ hoch, die Hantoberfläche beläuft sich auf $0,5046 \mathrm{~m}$.

\section{§ II. Stoffwechsel im Winter.}

Im Winter 1891-1892 bat Carletti ziemlich gearbeitet, der Knabe ist Schuster, die Mutter lag den bäuslichen Verrichtungen ob.

1) In dieser Periode gibt es keine Feldarbeit für Weiber und Knaben. 
1) Speisen, Zubereitung derselben, Vertheilung und Anstellung der Ve,rsuchsproben.

Die in den Versuchstagen genossenen Speisen waren Polenta, Suppe, Häring, Schweinsfett, Kastanienmehl. Die Polenta wird nach der auf unserem Lande herrschenden Gewohnheit aus Maismehl zubereitet, und wird gegessen entweder nachdem man sie in Schnitte zerlegt hat und etwas erkalten lässt, oder am frischen Fener geröstet. Die Suppe (Minestra) besteht aus Mehlteig und Bohnen, die in Wasser mit Zusatz von Schweinsfett und Zwiebel gekockt ist. Der Häring, wovon ein Stïck für eine Mahlzeit aller drei Personen ausreicht, wird im gerösteten Zustande gegessen. Das Schweinsfett, aus dem Abdomen entnommen, wird im Tiegel gekocht, wenn es als Zuspeis dienen soll, das Kastanienmehl wird auf die Polenta ausgebreitet und so ohne weiters genossen. Zur Bestimmung der Quantität der verschiedenen eingenommenen Speisen verwandten wir eine Wage von der Tragweite von 2 Kilo, und von der Empfindlichkeit für $5 \mathrm{egr}$. Man wog rasch die in Scheiben zerlegte, in der für jedes Individuum erforderlichen Menge gesammelte Polenta nahm gleichzeitig hievon eine Probe und führte sie in ein gewogenes mit einem emaillirten Stöpsel "versehenes”Gefäss ein. Die nach der Familiensitte in drei Näpfe vertheilte, wohl durchmischte Suppe wurde sofort gewogen. Die Probe wurde in ein Gefäss gebracht nach dem für die anderen warm zubereiteten Speisen üblichen Verfahren. Wir müssen hervorheben, dass die oben beschriebene Minestra nicht eine so bedeutende Quantität fliussigen Antheils enthält, wie jene, die in Italien gewöbnlich genossen wird, sondern viel dichter ist, sodass es viel leichter gelingt, sie gehörig zu durchrïhren und ein ziemlich homogenes Ganzes und zuverlässige Proben zu erhalten. - Zur Ermittlung: der von den drei Personen getrunkenen Wassermenge übergaben wir denselben ein Gefäss von bestimmter Capacität und merkten an, wie oft jedes Individuum Wasser geschöpft hatte.

2) Sammlung der Fäces und des Harns.

Da eine Versuchsperson unmittelbar vor Beginn der Versuche Stuhl gehabt und die anderen zwei ihre Faeces am Tage vorher entleert hatten, so musste man bei der Sammlung verschieden verfahren. Für den Mann galten als einer bestimmten 24 stündigen Nahrung entsprechende Faeces jene, welche mehrere Male während 
jener bestimmten Zeitperiode entleert worden waren, während für das Weib und den Knaben, welche ein einziges Mal ungefähr zu Mittag jedes Versuchstages Stubl hatten, als Auswurfsstoffe eines bestimmten Tages jene berechnet wurden, welche am folgenden Mittag entleert worden waren. Da die Diät vor Anstellung der Versuche ungefähr constant geblieben war, so war es überflüssig, mehrere Tage abzuwarten, ehe man die Experimente begann und ebenso unnütz war es, diese Versuche auf einen grösseren Zeitraum auszudehnen. Man bedenke ferner, dass wir ziemlich hartnäckigem Widerstande seitens der Individiuen selbst begegneten, welche selbst ohne Bildung und von ungebildeten Personen umgeben nur mit Widerwillen zu diesen Versuchen sich herbeiziehen liessen. Nur die Gewinnsucht der Leute und das Ansehen jenes Mannes ${ }^{1}$ ), der ihnen empfohlen hatte einzuwilligen, hatte sie endlich dazu bestimmt, als Versuchspersonen benutzt zu werden. Um die volle Zuverlässigkeit der Versuche zu sichern, bedienten wir uns der Beihillfe einer intelligenten Vertrauensperson, welche beauftragt war, ununterbrochen die drei Personen zu überwachen, damit nichts Abnormes stattfände, weder die Einführung von Speisen ausserhalb der festgestellten Mablzeiten, noch die Entleerung von Excrementen unter anderen Verhältnissen, als die von uns bestimmten. Dank dieser Vorsichtsmassregel gelang es, die Faeces, welche der Knabe am létzten Tage nicht in den Nachttopf, sondern auf dem Boden entleert hatte, zu sammeln. Obwohl dieselben geformt waren, war ein kleiner Verlust doch unvermeidlich. - Der Harn wurde von einem Morgen zum andern gesammelt und wie die Faeces in mit emaillirtem Stöpsel verschlossenen Gefässen aufgefangen und von einander durch bestimmte Zeichen unterschieden. Sofort nach der Sammlung des Harnes bestimmten wir das Gewicht, die Dichte und die Reaction desselben und berechneten dann aus Gewicht und Dichte das Volumen.

3) Stundeneintheilung des Tages, klimatische Verhältnisse, vollzogene Arbeit.

Die Versuche wurden am 3.4. 5. März 1892 ausgeführt, in welchen Tagen die mittlere Temperatur zwischen $-0,7$ and +1 ,

1) Der Besitzer des von ihnen bewohnten Hauses und der von ihnen bearbeiteten Aecker. 
der atmosphärische Luftdruck zwischen 752 und 758 schwankte. Am dritten März war es regnerisch, am 4. und 5. vollkommen unbewölkt. Am 3. und 4. dauerte die Tagesarbeit von 8-10, von 11 bis 2 Uhr Nachmittags und von 3 bis 4,30. Am 3. März bestand die erste Mahlzeit aus Polenta, Fett und Wasser, die zweite Mablzeit aus Polenta, Suppe und Wasser. Am 4. März war die erste Mahlzeit zusammengesetzt aus Polenta, Häring, Salat und Wasser, die zweite aus Polenta, Kastanienmehl und Wasser. Am 5. März war Rast, und die erste Mahlzeit bestand aus Polenta, Häring, Salat und Wasser die zweite aus Bohnensuppe, Polenta und Wasser. Der Gesundheitszustand der drei Versuchspersonen war vortrefflich, abgesehen von einer leichten Erkältung, die der Mann am 3. März wegen des regnerischen Wetters erfuhr.

4) Chemische Untersuchungsmethoden.

A. Speisen. Die Proben, welche in mit emaillirtem Stöpsel verschlossenen Gefässen aufbewahrt werden, sind gehörig geschnitten, im Mörser zerrieben, und in gewogenen Portionen 24 Stunden lang in einem Ofen bei $90^{\circ}$ trocknen gelassen. Der Rückstand wird im Mörser fein zerrieben und in gepaarte Uhrgläser gebracht, welche bis zum Gewichtsverluste im Essiccator unter der Wirkung von porösem Chlorcalcium ausgesetzt sind. Dieselbe Methode wurde auch für den Koth befolgt. An den so gewonnenen pulverförmigen Substanzen werden die Beistimmung des Stickstoffes, die Extraction der Fette und die Veraschung ausgefiuhrt.

Die erste Bestimmung geschieht nach der Kjeldahl'schen Methode, wie sie von $\mathrm{Ne} \mathrm{a} \mathrm{b} \mathrm{a} \mathrm{u} \mathrm{r} \mathrm{und} \mathrm{Voge}{ }^{1}$ \} beschrieben wird. Die Reagentien sind zuvor genau controlirt, die Vergleichsproben geben einen Fehler von $0,09-0,20 \%$ Stickstoff für $100 \mathrm{gr}$ Trockensubstanz. Die Extraction der Fette fand mit Apparaten statt, welche analog den von Medicus s${ }^{2}$ ) beschriebenen construirt sind. Die gepulverte Substanz wird in ein gewogenes Trockenfilter, welches in einem Glastrichter enthalten ist, gebracht. Der destillirte Aether fliesst fortwährend auf die zu extrahirende Substanz. Das Verfabren wird zum ersten Male 12 Stunden lang fortgesetzt, worauf

1) Neubauer und Vogel. Auflage 1890.

2) Lehmann K., Die praktische Hygiene S. 258. 1890. 
die entsprechenden Filter mit der Substanz bei $100^{\circ}$ getrocknet und wieder gewogen werden. Dann werden sie wieder drei oder vier Stunden lang extrahirt, getrocknet und wieder gewogen, und so fort bis zum Gewichtsverluste. Die Veraschung fand, wie gewöhnlich, in einem Platintiegel statt. Die Zahlen für Wasser, Stickstoff (als Eiweisssubstanz nach der jüngst von von Norden gemachten Angabe ungerechnet), Fette, Asche, pro mille frischer Substanz berechnet, werden addirt und ihre Summe von Tausend abgezogen stellt ungefähr die Menge der darin enthaltenen Kohlehydrate vor.

Behufs genauer Bestimmung des aus dem Abdomen entnommenen Schweinsfettes, italienisch "pancetta" genannt, welches ausser minimaler Fleischmenge eine grosse Quantität Fett enthält und daher bei der Trocknung nicht homogen bleibt, mussten wir eine gehörig durchrihhrte, getrocknete und abgewogene kleine Portion nehmen, auf dem Filter behufs Extraction des Fettes sammt dem Glase, anf dem "-.es aufbewahrt war, bringen, und nach Extraction der Fette den Stickstoff an einem bestimmten Theile des Riickstandes bestimmen. Die Analyse des Härings erfolgte nach Entfernung aller Gräten.

B. Faeces. Bezüglich der Faeces wurden dieselben Methoden, welche oben angeführt werden, verwendet, mit dem Unterschiede, dass vor Entnahme bestimmter Portionen aus den Sammelgefässen die Substanz mit einem Porzellanlöffel längere Zeit durchrührt wurde. - Auch die geformten Faeces waren stets in dem Grade weich, dass immer eine gehörige Durchmischung gelang.

C. Harn. Wie gesagt wurde der Harn nach Ablauf von 24 Stunden gesammelt, sofort in den entsprechenden Gefässen gewogen und auf Reaction und Dichte geprifft. Die Stickstoffbestimmung geschah nach der oben erwähnten Methode, die Bestimmung des Harnstoffes nach einer von Professor P. Al b e r to n i modificirten Methode, welche seit längerer Zeit in unserem Laboratorium iiblich ist. Das Wesen der Modification besteht darin, dass man mit dem Quecksilber in einer Gazburette experimentirt, welche oben mit einem Hahne ausgestattet ist. Man füllt den Harn durch den Hahn ein, welcher dann nicht mehr angerührt wird, liest die Quantität eingegossenen Harnes ab, macht die Correction des Meniscus und setzt von unten durch die Burette Natriumhypobromid zu. Der Vortheil dieser Methode liegt darin, dass man das Quecksilber mit dem 
Ueber die Nahrungs- und Stoffwechselbilanz des italienischen Bauers. 223

Harne nicht in Berihhrang bringt und daher keine Harnmenge der Messung sich entzieht.

5) Bestandtheile der Speisen.

Unter Befolgung der erwähnten Massregeln haben wir die Zahlen erhalten, welche in der Tabelle I angeführt sind. Wir miissen bemerken, dass von den Bestandtheilen der Speisen direct nur der Stickstoff, die in Aether löslichen Substanzen und die Asche bestimmt wurden.

Aus dem Stickstoffe berechneten wir nach der jüngst gemachten Angabe ( on Noorden) die Eiweissstoffe, indem wir die für den Stickstoff ermittelte Zahl mit 6,25 multiplicirten. Diese Bestimmung: hatte lediglich den $Z_{w e c k}$, daraus jene der Kohlenhydrate abzuleiten.

Als Fett haben wir im Einklange mit den meisten Forschern alle jene Substanzen angeseben, welche in Aether löslich und mit demselben extrahirbar sind.

Was die mineralischen Bestandtheile anbelangt, glaubten wir, keinen allzugrossen Fehler zu begehen, wenn wir sie einfach in Aschenform bestimmten.

Dies musste dazu dienen, eine Summe von Fett, Eiweissstoffen und mineralischen Substanzen zu bilden, welche von der Quantität Trockenrückstand abgezogen, annähernd die Zahl Kohlenhydrate liefern sollte.

Tabelle I. Zusammensetzung der Nahrungsmittel pro Mille.

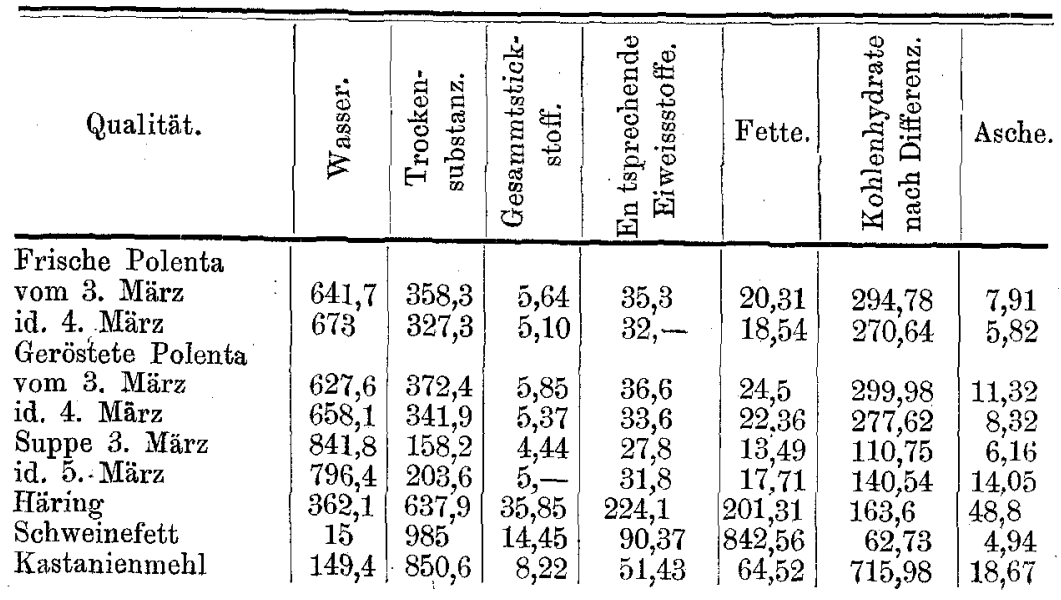


6) Eingeführte $\mathrm{Speisen} u n d \mathrm{Nährstoffe.}$

Wir fassen dieselben in der beigegebenen Tabelle II zusammen.

Tabelle II. Nahrung des Mannes.

\begin{tabular}{|c|c|c|c|c|c|c|c|c|c|}
\hline \multirow{2}{*}{ Datum. } & \multicolumn{2}{|l|}{ Speisen. } & \multirow{2}{*}{ 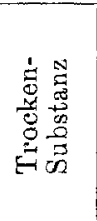 } & \multirow{2}{*}{ 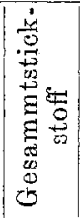 } & \multirow{2}{*}{ 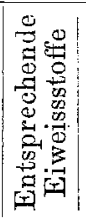 } & \multirow{2}{*}{ 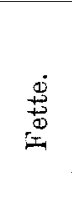 } & \multirow{2}{*}{ 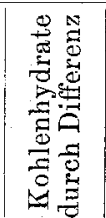 } & \multirow{2}{*}{$\begin{array}{l}0 \\
\text { 옹 } \\
\text { प }\end{array}$} & \multirow{2}{*}{ 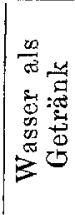 } \\
\hline & Qualität & $\begin{array}{l}\text { Quan- } \\
\text { tität }\end{array}$ & & & & & & & \\
\hline \multirow[t]{2}{*}{ 3. März } & $\begin{array}{l}\text { Polenta \{ frische } \\
\text { Schweinefett } \\
\text { Suppe }\end{array}$ & $\begin{array}{r}1165 \\
350 \\
37 \\
790\end{array}$ & $\begin{array}{c}417,42 \\
130,34 \\
36,45 \\
125\end{array}$ & $\begin{array}{l}6,57 \\
2,04 \\
5,32 \\
3,51\end{array}$ & $\begin{array}{r}41,12 \\
12,81 \\
3,33 \\
21,96\end{array}$ & $\begin{array}{r}23,66 \\
8,57 \\
31,17 \\
10,65\end{array}$ & $\begin{array}{r}105 \\
343,43 \\
1,77 \\
87,53\end{array}$ & $\begin{array}{l}3,96 \\
9,21 \\
0,18 \\
4,86\end{array}$ & \multirow{2}{*}{1410} \\
\hline & & $\overline{2342}$ & 709,21 & $\overline{17}, \overline{44}$ & 79,25 & $\overline{74}, \overline{05}$ & 537,73 & $\overline{18}, 21$ & \\
\hline \multirow[t]{2}{*}{ 4. März } & $\begin{array}{l}\text { Polenta }\left\{\begin{array}{l}\text { frische } \\
\text { geröstete }\end{array}\right. \\
\text { Häring } \\
\text { Kastanienmehl }\end{array}$ & $\begin{array}{c}1009 \\
1024 \\
32 \\
71,5\end{array}$ & $\begin{array}{r}375,77 \\
334,85 \\
20,42 \\
60,82\end{array}$ & $\begin{array}{l}5,24 \\
5,90 \\
1,14 \\
0,58\end{array}$ & $\begin{array}{r}32,78 \\
36,92 \\
7,17 \\
3,67\end{array}$ & $\begin{array}{r}24,72 \\
18,98 \\
6,44 \\
4,61\end{array}$ & $\begin{array}{r}316,85 \\
272,99 \\
5,23 \\
51,21\end{array}$ & $\begin{array}{r}5,96 \\
11,42 \\
1,56 \\
1,33\end{array}$ & 1750 \\
\hline & & 2136,5 & $\overline{791,86}$ & $\overline{12, \overline{86}}$ & $80, \overline{54}$ & $\overline{54,75}$ & $\overline{646,28}$ & $20, \overline{27}$ & \multirow{3}{*}{1000} \\
\hline \multirow[t]{2}{*}{ 5. März } & $\begin{array}{l}\text { Geröstete Polenta } \\
\text { Häring } \\
\text { Suppe }\end{array}$ & $\begin{array}{r}1621 \\
33 \\
866\end{array}$ & $\begin{array}{r}525,27 \\
21,06 \\
178,52\end{array}$ & $\begin{array}{l}8,71 \\
1,18 \\
4,33\end{array}$ & $\mid \begin{array}{r}54,45 \\
7,39 \\
27,10\end{array}$ & $\begin{array}{r}31,22 \\
6,64 \\
15,33\end{array}$ & $\begin{array}{r}420,12 \\
5,39 \\
125,93\end{array}$ & $\begin{array}{r}13,48 \\
1,61 \\
12,16\end{array}$ & \\
\hline & & $\overline{2520}$ & 724,85 & 14,22 & 88,94 & $\overline{63}, 1 \overline{9}$ & $\overline{551,44}$ & 27,25 & \\
\hline
\end{tabular}

Tabelle III. Ernähruug des Weibes.

\begin{tabular}{|c|c|c|c|c|c|c|c|c|c|}
\hline \multirow{2}{*}{ Datum } & \multicolumn{2}{|l|}{ Speisen } & \multirow{2}{*}{ 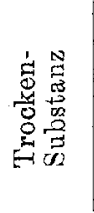 } & \multirow{2}{*}{ 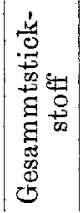 } & \multirow{2}{*}{ 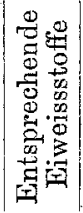 } & \multirow{2}{*}{$\begin{array}{l}0 \\
\pm 0 \\
0 \\
0.1\end{array}$} & \multirow{2}{*}{ 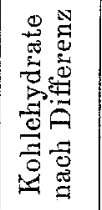 } & \multirow{2}{*}{$\frac{0}{0}$} & \multirow{2}{*}{ 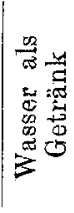 } \\
\hline & Qualität & $\begin{array}{l}\text { Quan- } \\
\text { tität }\end{array}$ & & & & & & & \\
\hline \multirow{5}{*}{ 3. März } & Polanto I frisch & 755,5 & 270,7 & 4,26 & 26,66 & 15,34 & 223,73 & 5,97 & \multirow{5}{*}{750} \\
\hline & Polenta geröstet & 259 & 96,46 & 1,51 & 9,47 & 6,34 & 77,72 & 2,93 & \\
\hline & Schweinefett & 19 & 18,72 & 0,27 & 1,71 & 16 & 0,92 & 0,09 & \\
\hline & Suppe & 810 & 128,15 & 3,60 & 22,51 & 10,92 & 89,74 & & \\
\hline & & 1843,5 & $514, \overline{03}$ & 9,64 & 60,35 & 48,60 & 392,11 & $\overline{1} \overline{3}, \overline{97} \mid$ & \\
\hline \multirow{4}{*}{ 4. März } & Polenta geröstet & 925 & 344,47 & 5,41 & 33,85 & 22,66 & 277,49 & 10,47 & \multirow{3}{*}{1150} \\
\hline & Härinore frisch & $\begin{array}{r}945 \\
32\end{array}$ & $\left|\begin{array}{r}309,02 \\
20,42\end{array}\right|$ & $\begin{array}{l}4,83 \\
1,14\end{array}$ & $\mid \begin{array}{r}30,24 \\
7,17\end{array}$ & {$\left[\begin{array}{r}1,52 \\
6,44\end{array}\right.$} & $\left|\begin{array}{r}250,76 \\
5,23\end{array}\right|$ & $\begin{array}{r}5,5 \\
1,56\end{array}$ & \\
\hline & Kastanienmehl & 71,5 & 60,82 & 0,58 & 3,67 & \begin{tabular}{|l|}
$4,7 x$ \\
4,61
\end{tabular} & 51,21 & 1,33 & \\
\hline & & 1973,5 & $\overline{734,73}$ & $\overline{11,96}$ & $\overline{74,93}$ & $51, \overline{23}$ & $\overline{589,69}$ & $\overline{18,84}$ & \multirow{5}{*}{1120} \\
\hline \multirow{4}{*}{ 5. März } & Geröstete Polenta & 1263 & 431,83 & 6,78 & 42,43 & $23,41\}$ & 365,49 & 10,5 & \\
\hline & Häring & 30 & 19,14 & 1,07 & 6,72 & $\begin{array}{r}6,03 \\
\end{array}$ & $\begin{array}{c}4,9 \\
4\end{array}$ & 1,46 & \\
\hline & & 2125 & $\frac{109, \pm}{620,37}$ & $\left|\frac{4,0}{12,01}\right|$ & 75,19 & $\frac{14,73}{44,17}$ & $\left|\frac{116,94}{487,39}\right|$ & $\left|\begin{array}{l}11,69 \\
23,65\end{array}\right|$ & \\
\hline & & 2120 & & & {$[0,10$} & $4 \pi, 16$ & 100,900 & & \\
\hline
\end{tabular}


Tabelle IV. Ernährung des Knaben.

\begin{tabular}{|c|c|c|c|c|c|c|c|c|c|}
\hline \multirow{2}{*}{ Datum } & \multicolumn{2}{|l|}{ Speisen } & \multirow{2}{*}{ 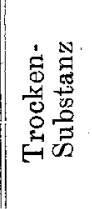 } & \multirow{2}{*}{ 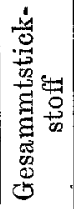 } & \multirow{2}{*}{ 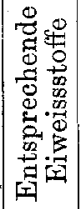 } & \multirow{2}{*}{ 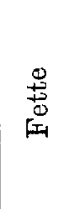 } & \multirow{2}{*}{ 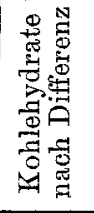 } & \multirow{2}{*}{$\begin{array}{l}\frac{0}{0} \\
\frac{0}{4}\end{array}$} & \multirow{2}{*}{ 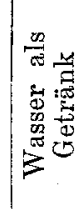 } \\
\hline & Qualität & $\begin{array}{l}\text { Quan- } \\
\text { tität }\end{array}$ & & & & & & & \\
\hline \multirow{5}{*}{ 3. März } & Polents f frisch & 224 & 80,26 & 1,26 & 7,90 & 4,54 & 66,03 & 1,77 & \multirow{5}{*}{430} \\
\hline & Polenta geröstet & 239 & 89,01 & 1,39 & 8,74 & 5,85 & 71,69 & 2,70 & \\
\hline & Schweinefett & 20 & 19,7 & 0,28 & 1,80 & 16,85 & 0,95 & 0,09 & \\
\hline & Suppe & 720 & 113,91 & 3,20 & 20,01 & 9,71 & 79,76 & 4,43 & \\
\hline & & 1203 & 302,88 & 6,13 & $\overline{38,45}$ & $\overline{36,95}$ & $\overline{218,43}$ & 8,99 & \\
\hline \multirow{5}{*}{ 4. März } & Polenta \{ geröstet & 550 & 204,82 & 3,22 & 20,13 & 13,47 & 166 & 6,22 & \multirow{4}{*}{890} \\
\hline & Fotenta friseh & 613 & 200,46 & 3,13 & 19,61 & 11,36 & 165,93 & 3,56 & \\
\hline & Häring. & 21 & 13,4 & 0,75 & 4,7 & 4,22 & 3,43 & 1,02 & \\
\hline & Kastanienmehl & $\frac{71, b}{10655}$ & $\frac{60,82}{470,50}$ & $\frac{0,58}{7,88}$ & $|3,67|$ & $\frac{4,61}{23,66}$ & $\frac{51,21}{28657}$ & $\mid \begin{array}{l}1,33 \\
197^{6} \\
\end{array}$ & \\
\hline & 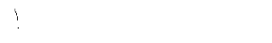 & & 48,00 &, 00 & $\mid 40,11\}$ & 03,00 & (2006, & {$[12,15 \mid$} & \multirow{4}{*}{800} \\
\hline \multirow{3}{*}{ 5. März } & Geröstete Polenta & 1026 & 350,8 & 5,51 & 34,46 & 22,93 & 284,88 & 8,53 & \\
\hline & Häring & $\begin{array}{r}25 \\
796\end{array}$ & $\begin{array}{c}15,95 \\
162,1\end{array}$ & $\begin{array}{l}0,89 \\
6,39\end{array}$ & $\begin{array}{c}5,6 \\
24,91\end{array}$ & $\begin{array}{r}5,03 \\
14,09\end{array}$ & $\begin{array}{r}4,09 \\
11192\end{array}$ & $\begin{array}{r}1,22 \\
11,18\end{array}$ & \\
\hline & & 1847 & $5 \overline{28,85}$ & $\longdiv { 1 2 , 7 9 }$ & $\overline{64,97}$ & $\overline{42,05}$ & $\overline{400,89}$ & 20,93 & \\
\hline
\end{tabular}

7) Ausscheidung mit dem Kothe.

Tabelle V enthält alle Zahlen, welche dieser Ausscheidung bei den drei Versuchspersonen entsprechen.

Diese Ausscheidung stellt zum grössten Theile einen Verlust an nicht absorbirtem Nährmaterial vor und zum minimalen Theile stammt sie ans Epithelien, Schleim, Verdaunngssäften etc.

Bei der Berechnung aller Bestandtheile befolgten wir die bereits in 5) für die Speisen angegebenen Regeln; doch mïssen wir hervorheben; dass nicht alle in den Faeces gefundenen Mineralsubstanzen aus der Nahrung stammen, sondern ausser jenem Theile, welcher von dem Verluste des Magendarmkanales herrihrt, giebt es einen Theil, welcher seinen Ursprung dem als Getränk eingeführten Wasser verdankt.

Diese Thatsache kann nur minimale Differenzen herbeiführen, weil das getrunkene Wasser nur ungefähr 1 Gramm Trockensubstanz pro Liter enthält. 
Tabelle V. Ausscheidung mit den Faeces.

\begin{tabular}{|c|c|c|c|c|c|c|c|c|}
\hline Datum & 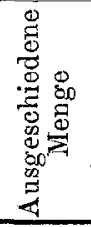 & 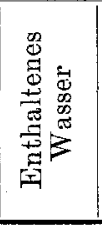 & 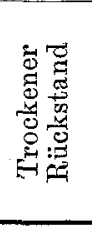 & 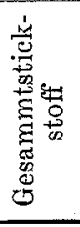 & 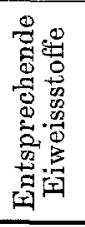 & 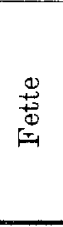 & 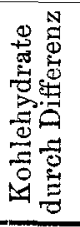 & 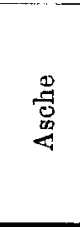 \\
\hline \multirow{3}{*}{ 3. März } & 613,6 & 515,16 & 98,44 & $4, \mathbf{1 6 3}$ & 29,76 & 9,65 & 57,75 & 1,28 \\
\hline & 205,3 & 155,51 & 49,79 & 2,245 & 14,03 & 6,55 & 21,87 & 7,33 \\
\hline & 170,25 & 128,39 & 41,86 & 0,498 & 3,08 & 5,17 & 27,35 & 6,26 \\
\hline \multirow{3}{*}{ 4. März } & 395,8 & 333,92 & 61,88 & 3,048 & 19,05 & 1,43 & 33,6 & 7,80 \\
\hline & 239 & 184,09 & 54,91 & 2,243 & 14,01 & $1,-$ & 31,86 & 8,04 \\
\hline & 105,5 & 80,24 & 25,26 & 1,145 & 7,15 & 2,47 & 12,59 & 3,05 \\
\hline \multirow{3}{*}{ อ. März } & 405,2 & 341,36 & 63,84 & 1,664 & 10,40 & 6,83 & 37,76 & 8,85 \\
\hline & 303,1 & 234,97 & 68,13 & 3,062 & 19,13 & 8,19 & 30,70 & 10,11 \\
\hline & 77,55 & 59,89 & 17,66 & 0,838 & 5,23 & 7,67 & 2,17 & 2,59 \\
\hline
\end{tabular}

8) A usscheidung mit dem Harne.

Der mit dem Harne ausgeschiedene Stiekstoff ist der treueste Repräsentant der Zersetzung der in den Organismus eingeführten Eiweisssubstanzen und des inneren Stoffwechsels der Gewebe. Berechnet man die Ziffer des Harnstoffes und des Stickstoffes, welcher dem ersteren entspricht und zieht man dieselbe von der Gesanmtstickstoffzahl ab, so bleibt jener Theil Stickstoff iubrig, welcher aus den anderen stickstoffhaltigen Substanzen, die neben dem Harnstoffe im Harne enthalten sind, wie Harnsäure, Kreatinin, Kreatin, Xanthin, Hypoxanthin.

Ferner haben wir uns eine Vorstellung bilden wollen von den Verwesungsprocessen, welche im Darmkanale sich abspielen, und in derselben folgenden Tabelle führen wir die Ziffern an, welehe der präformirten und der gepaarten Schwefelsäure entsprechen.

Bezuiglich abnormer Harnbestandtheile müssen wir hervorheben, dass in unserem Falle nie weder Zucker noch Eiweiss angetroffen wurden.

Die Reaction des Harnes war stets sauer. 
Ceber die Nahrungs- und Stoffwechselbilanz, des italienischen Bauers. 227 Tabelle VI. Ausscheidung mit dem Harne.

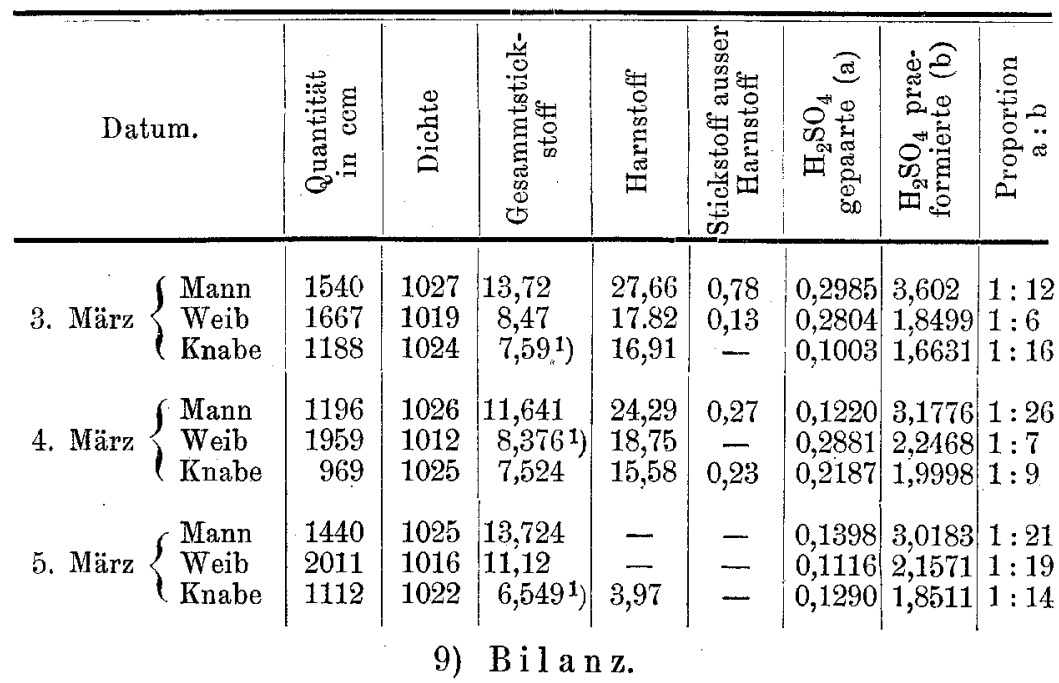

Wenn wir jetzt die Quantität eingeführter Nährstoffe mit jener der durch den Harn ausgeschiedenen und mit der Menge der im Organismus zerstörten und im Harne erschienenen Eiweissstoffe in Beziehung bringen, so werden wir die Materialien finden, welche assimilirt oder ausgenuitzt worden sind.

Tabelle VII, VIII, IX fassen eben die Zahlen zusammen, welche sich auf den Stickstoff, die Fette, die Kohlenhydrate und das Wasser beziehen.

Tabelle VII. Sticks toffbilanz.

\begin{tabular}{|c|c|c|c|c|c|c|}
\hline Datum & Speise & Faeces & $\begin{array}{l}\text { Speise- } \\
\text { Faeces }\end{array}$ & Harn & $\begin{array}{l}\text { Diffe- } \\
\text { renz }\end{array}$ & $\begin{array}{l}\text { id. berechnet } \\
\text { auf } \\
\text { Albuminoide }\end{array}$ \\
\hline 3. März $\left\{\begin{array}{l}\text { Mann } \\
\text { Weib } \\
\text { Knabe }\end{array}\right.$ & $\begin{array}{r}12,67 \\
9,65 \\
6,16\end{array}$ & $\begin{array}{l}4,76 \\
2,24 \\
0,49\end{array}$ & $\begin{array}{l}7,91 \\
7,41 \\
5,67\end{array}$ & $\begin{array}{r}13,72 \\
8,47 \\
7,59\end{array}$ & $\begin{array}{l}-5,81 \\
-1,06 \\
-1,92\end{array}$ & $\begin{array}{l}-36,31 \\
-\quad 6,62 \\
-12\end{array}$ \\
\hline 4. März $\left\{\begin{array}{l}\text { Mann } \\
\text { Weib } \\
\text { Knabe }\end{array}\right.$ & $\begin{array}{r}12,88 \\
11,98 \\
7,69\end{array}$ & $\begin{array}{l}3,04 \\
2,24 \\
1,14\end{array}$ & $\begin{array}{l}9,84 \\
9,74 \\
6,55\end{array}$ & $\begin{array}{r}11,64 \\
8,37 \\
7,52\end{array}$ & $\begin{array}{l}-1,8 \\
+1,37 \\
-0,97\end{array}$ & $\begin{array}{r}11,25 \\
+\quad 8,56 \\
-\quad 6,06\end{array}$ \\
\hline ๖. März $\left\{\begin{array}{l}\text { Mann } \\
\text { Weib } \\
\text { Knabe }\end{array}\right.$ & $\begin{array}{l}14,23 \\
12,03 \\
10,40\end{array}$ & $\begin{array}{l}1,66 \\
3,06 \\
0,83\end{array}$ & $\begin{array}{r}12,57 \\
8,97 \\
9,57\end{array}$ & $\begin{array}{r}13,72 \\
11,12 \\
6,54\end{array}$ & $\begin{array}{l}-1,15 \\
-2,15 \\
+3,03\end{array}$ & $\begin{array}{r}-7,18 \\
-13,43 \\
+18,93\end{array}$ \\
\hline
\end{tabular}

1) Stickstoff des Harnstoffes. Die Bestimmung des Gesammtstickstoffe ging verloren. 
Tabelle VIII.

\begin{tabular}{|c|c|c|c|c|c|c|c|}
\hline \multicolumn{8}{|c|}{$\mathrm{B}$ i l a n $\mathrm{z}$} \\
\hline \multicolumn{4}{|c|}{ derFette } & \multicolumn{2}{|c|}{ und der } & \multicolumn{2}{|c|}{ Kohlehydrate } \\
\hline Dat & & Speise & Faeces & $\begin{array}{c}\text { Aus- } \\
\text { genützt }\end{array}$ & Speise & Faeces & $\begin{array}{l}\text { Aus- } \\
\text { genützt }\end{array}$ \\
\hline \multirow{3}{*}{ 3. März } & Mann & 74,05 & 9,65 & 64,40 & 537,73 & 57,75 & 479,98 \\
\hline & Weib & 48,6 & 6,55 & 42,05 & 392,11 & 21,87 & 370,24 \\
\hline & Knabe & 36,95 & 5,17 & 31,78 & 218,43 & 27,35 & 191,08 \\
\hline \multirow{3}{*}{ 4. März } & Mann & 56,75 & 1,43 & 55,32 & 644,28 & 33,6 & 610,68 \\
\hline & Weib & 51,23 & $1,-$ & 50,23 & 589,67 & 31,86 & 557,83 \\
\hline & Knabe & 33,66 & 2,47 & 31,19 & 386,57 & 12,59 & 373,98 \\
\hline \multirow{3}{*}{ 5. März } & Mann & 59,79 & 6,83 & 52,36 & 551,44 & 32,58 & 518,86 \\
\hline & Weib & 44,77 & 8,19 & 35,98 & 487,33 & 23,57 & 403,76 \\
\hline & Knabe & 42,05 & 7,67 & 34,38 & 400,89 & 0,27 & 400,62 \\
\hline
\end{tabular}

Tabelle IX. Wasserbilanz.

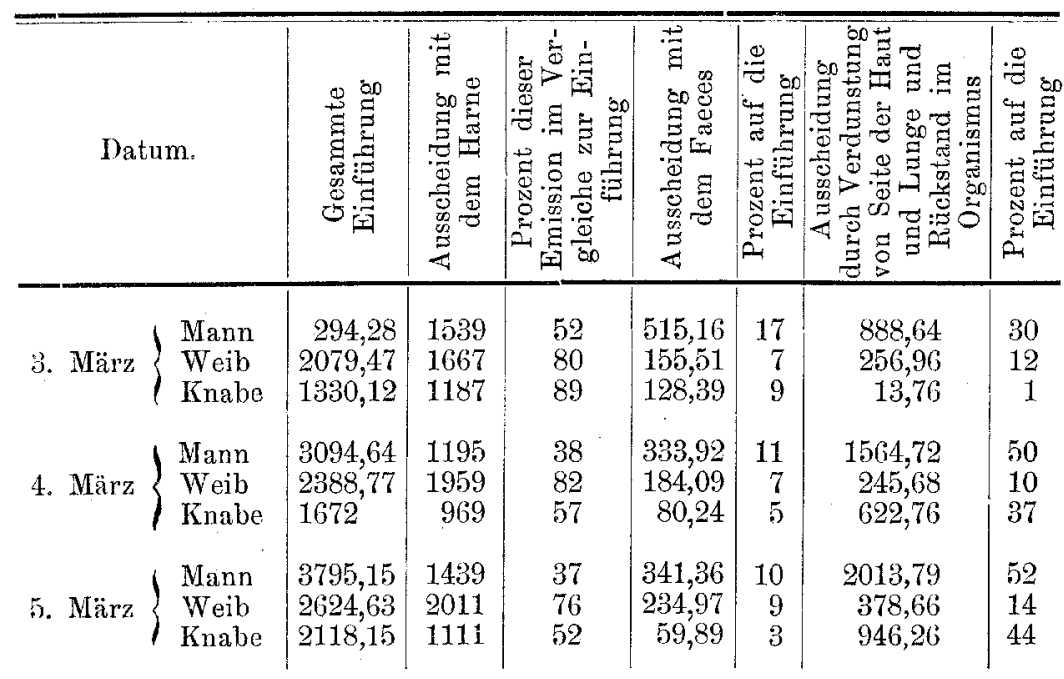

10) Procentgebaltan Stickstoff, Fett, Koblenhydraten in den Faees.

Es ist wichtig zu erfahren, wie viel von den eingeführten Nährstoffen ungeändert den Magendarmkanal passirt hat, oder besser gesagt wie viel Stickstoff, Fett und Kohlenhydrate durch das Darmrohr verloren gegangen und in den Faeces ersehienen sind. 
Ueber die Nahrungs- und Stoffwechselbilanz des italienischen Bauers. 229

$\mathrm{Zu}$ diesem Zwecke haben wir die Tabelle $\mathrm{X}$ zusammengestellt, welcher ersichtlich ist, wie viel Substanzen pro 100 eingeführte Theile entleert wurden.

Tabelle X. Prozentgehalt an Stickstoff, Fett und Kohlehydraten in den Faeces.

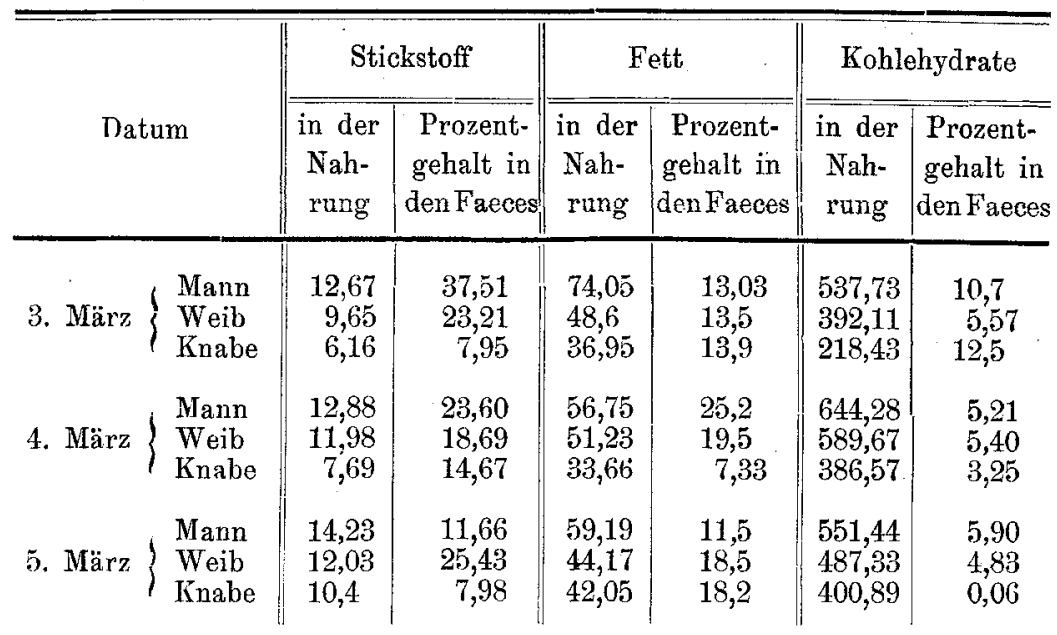

\section{§III. Stoffwechsel im Sommer.}

August 1892.

1) Versuchspersonen. Es handelt sich um die bereits erwähnten drei Individuen; dieselben erfreuten sich von März bis August ausgezeichneter Gesundheit, arbeiteten wie gewöhnlich mit sehr kurzen Unterbrechungen, weil die gute Jahreszeit eine fast unterbrochene Beschäftigung von Feldarbeiten aller Art gestattete. Das Körpergewicht aller drei Personen bewegte sich ungefähr innerbalb derselben Grenzen, welche im vergangenen März verzeichnet worden waren.

2) Spe is e n. Zum Unterschiede von der im Winter verbrauchten Nahrung, besteht dieselbe im Sommer im Wesentlichen aus Brot und Suppe. Das Brod wird aus Weizenmehl gebacken, von welchem nur die grobe Kleie entfernt wird, während die feine, italienisch "tritello" belassen wird. Das Brod bildet Scheiben zu Paaren von 150 Gr. Durchschnittsgewichte. Der ungekochte Teig ist ziemlich hart und enthält sehr wenig Sauerteig. Unserem Gaumen schmeckt das Brot gut, gesalzen, wie überhaupt das Brot der 
Provinz Ferrara und nur am dritten Tage im Sommer ist es etwas hart, aber noch immer geniessbar. Die Suppe ist die gewöhnliche Winterminestra, besteht aus Mehlteig und Bohnen, welche in der oben angegebenen Weise gekocht sind. Entsprechend den accessorischen Speisen, welche bei der Winternahrung besprochen wurden, giebt es im Sommer andere specielle Nahrungsmittel, wie Käse, Wassermelone, and Thunfisch in Oel.

Diese und andere Speisen, die in unserem Falle nicht genossen wurden, repräsentiren einen minimalen Antheil von geringer Bedeutung in der Einnahmebilanz, wie man aus den entsprechenden Tabellen ersehen wird. Besondere Erwähnung verdient die Wassermelone, welche in beträchtlicher Menge eingeführt wird, aber wegen des minimalen Gehaltes an festen Bestandtheilen keinen erheblichen Einfluss auf den Stoffwechsel ausüibt, abgesehen vielleicht von der Wirkung, die das darin enthaitene Wasser entfaltet. Mit der Wassermelone assen unsere Bauern viel Brot und stillten rascher und in schmackhafterer Weise ibren Durst.

3) Bei der Sammlang von Versuchproben des Brotes und der Melone verfubren wir folgendermassen : für Thunfisch und Käse war das Verfahren das gewöhnliche.

Die Substanz wurde sofort in ein mit einem emaillirten Stöpsel verschlossenes Gefäss eingeführt. Am ersten Tage wurden einige Brotscheiben gewogen und am letzten wieder gewogen. Der Unterschied war so unerheblich, dass er nicht berïcksichtigt zu werden brauchte. Einige Stiucke sofort in die gewöhnlichen Gefässe gebracht und mit Theer verschlossen bildeten die Proben für die quantitative Untersuchung. Für die Bestimmung wurden die Stïcke herausgenommen, im Mörser zerrieben, von Nenem in das Gefäss eingeführt, damit sie den eventuell zurückgebliebenen Wasserdampf absorbiren könnten, und Portionen davon für die verschiedenen Analysen entnommen. Was die Melone betrifft, genossen natürlich alle drei Personen dieselbe Frucht. Die Samen wurden aufbewahrt und sammt der Hiulle von dem Gewichte der jedem Individuum übergebenen Portion abgezogen. Die Versuchsportion wurde sofort von dem Samen und der Hïlle befreit und in das Gefäss eingeführt. Für die Bestimmung wurde sie in toto verwendet, indem mehrere Male das Gefäss mit Wasser vollständig ausgespiult wurde.

4) Bei der Sammlung der Excrete befolgten wir die 
Ueber die Nahrungs- und Stoffwechselbilanz des italienischen Bauers, 231

oben beschriebenen Massregeln. Die gesammelten Faeces waren mindestens 12 Stunden nach Beginn des Versuches und 24 Stunden nach der letzten vorausgegangenen Mahlzeit entleert.

5) Stundeneintheilung. Klimatische Verhältnisse, a us geführte Arbeit und Mahlzeiten. Die Versuche fanden am 13. 14. 15. August 1892 statt, wovon zwei Arbeitstage und ein Ruhetag waren. Die durchschnittliche Temperatur der drei Tage schwankte zwischen +24 und $+25,7$, der Luftdruck zwischen 764,5 u.764,7. Der Himmel warimmer unbewölkt. Am 13. und 14. begann die Arbeit um $4 \mathrm{Uhr}$ in der Früh und bestand in der Bearbeitung des Hanfes. Um 8,30 fand die erste Mahlzeit statt, welche aus Brot, Thun und Wasser bestand, von 9,30 bis 2 Uhr wurde die Arbeit rom Manne und Knaben wieder aufgenommen. Um 2 Uhr fand die zweite Mahlzeit statt, welche aus Minestra mit Bohnen, Brot und Wasser bestand. Von 2 bis $8 \mathrm{Uhr}$ wurde wieder an die Arbeit geschritten, während welcher die Familie etwas Brot ass und viel Wasser trank, wie in der Tabelle angeführt ist. Um 8 Uhr letzte. Mahlzeit, die aus Thunfisch, Käse oder Wassermelone und Brot bestand.

Der Gesundheitszustand aller drei Versuchṣpersonen war aus: gezeichnet, die Arbeit des Weibes war etwas weniger mühsam, wegen der häuslichen Verrichtungen.

6) Chem ische Untersuchungsmethoden. Ausser den Versuchen, welehe wir bereits bei Besprechung der Winterbilanz besprochen baben, mussten wir hier uns mit anderen Bestimmungen befassen, welche sich auf die drei neuen im Sommer eingenommenen Speisen bezogen und zwar Brot an Stelle der Polenta, Käse und Thunfisch an Stelle des Schweinsfettes und Melone an Stelle des Kastanienmehles.

Für das Brot befolgten wir dieselbe Methode, wie für die Polenta nach der Trocknung. Bezigglich des Käses beriicksichtigten wir den Umstand, dass in demselben in mehr oder minder erheblicher Quantität Stickstoffsubstanzen enthalten sein können, welche nicht Eiweisskörper und mit Aether extrahirbar sind, dass daher bei der Umrechnung der Ziffer des Gesammtstickstoffes in Eiweisskörper in toto, man eine Menge Albuminoide erhalten hätte, welche die Norm übersteigt und im Wesentlichen eine Fehlerquelle abgegeben hätte bei Berechnung der Kohlenhydrate, die durch Differenz bestimmt werden. Diese Erwägung bewog uns mit Aether 
den trockenen Käse zu extrahiren und aus dem entfetteten Rüickstande den Stickstoffgehalt $\mathrm{zu}$ bestimmen. Indem wir auch den Gesammtstickstoff für die Körperbilanz in Rechnung zogen, fanden wir Zahlen, welche mit den bis jetzt bekannten im Einklange stehen und welche vielleicht für die Fette um ein Geringes den thatsächlichen Gehalt übertreffen. Dasselbe Verfahren hätten wir gerne eingeschlagen bei dem Thunfische unter Oel, welcher bestimmt stickstoffhaltige Substanzen enthält, welche aus Eiweisskörpern stammen. In den für dieses Nahrungsmittel ermittelten Werthen, welche übrigens mit den bis dato gefundenen übereinstimmen, muss eine Quantität Kohlenhydrate repräsentirt sein, welche niedriger, und eine Eiweissmenge, welche höher ist als gewöhnlich. Indess ist es ansgemacht, dass diese Unterschiede in unserem Falle keine wesentliche Bedeutung haben, weil diese Nahrungsmittel von allen drei Versuchspersonen in kleinen Mengen genossen wurden.

Bezüglich der Melone verfuhren wir nicht nach einer besonderen Methode. Die Trocknung fand da langsamer statt, weil eine grössere Wassermenge verdunsten musste, aber in feiner Pulverform zerrieben war die Substanz für die gewöhnlichen Versuche vortrefflich geeignet.

In der Tabelie XI führen wir die Zahlen an, welche wir auf Grund der an den Proben der einzelnen eingeführten Nahrungsmittel angestellten Untersuchungen gewonnen haben.

Tabelle XI. Zusammensetzung der Nahrungsmittel pro Mille.

\begin{tabular}{|c|c|c|c|c|c|c|c|}
\hline Qualität & 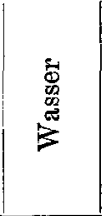 & 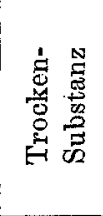 & 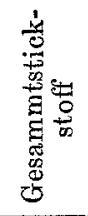 & 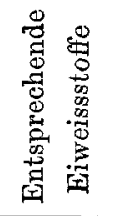 & Fette & 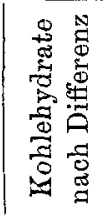 & Asche \\
\hline Brot 13. August & 202,1 & 797,9 & 17,8 & 111,48 & 15,79 & 659,17 & 11,46 \\
\hline Suppe $\quad$ (13. Aug. & 712,3 & 287,7 & 8,5 & อ๊ 3,18 & 41,25 & 179.05 & 14,22 \\
\hline Mehlteig 14 . Aug. & 793, & 206,4 & 5,9 & 37,08 & 20,45 & 135,11 & 13,76 \\
\hline u. Bohnen 15. Aug. & 794,5 & 205,5 & 7,4 & 46,74 & 31,99 & 107,93 & 18,84 \\
\hline Parmesankäse & 335,3 & 664,7 & 50,5 & $\left.269,2^{1}\right)$ & 312,6 & 43,32 & 41,58 \\
\hline Thunfisch (in Oel) & 474,7 & 525,9 & 43,2 & 270,1 & 243,3 & $1,-$ & 11,5 \\
\hline Melone (ohne Samen) & 986,49 & 13,51 & 0,39 & 2,45 & 3,59 & 5,72 & 1,75 \\
\hline
\end{tabular}

1) Die aus dem Gesammtstickstoffe abgeleiteten Eiweissstoffe des Käses würden 315,8 sein; indem aber wir eingedenk waren, dass unter den als Fett 
Uéber die Nahrungs und Stoffwechselbilanz des italienischen Bauers. 233

7) Eingeführte $\mathrm{Nahrungsmittel.} \mathrm{Für} \mathrm{den} \mathrm{Mann}$ sind sie zusammengefasst in Tabelle XII.

Für das Weib sind sie angeführt in Tabelle XIII.

Die Nahrung des Knaben ist beschrieben in Tabelle XIV.

Tabelle XII. Einfuhr beim Manne.

\begin{tabular}{|c|c|c|c|c|c|c|c|c|c|}
\hline \multirow{2}{*}{ Datum } & \multicolumn{2}{|c|}{ Speisen } & \multirow{2}{*}{ 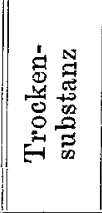 } & \multirow{2}{*}{ 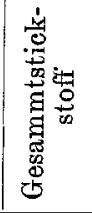 } & \multirow{2}{*}{ 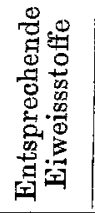 } & \multirow{2}{*}{ 売 } & \multirow{2}{*}{ 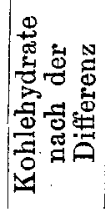 } & \multirow{2}{*}{$\begin{array}{l}\frac{0}{8} \\
\frac{0}{4}\end{array}$} & \multirow{2}{*}{ 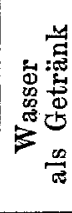 } \\
\hline & Qualität & $\begin{array}{c}\text { Quan- } \\
\text { tität }\end{array}$ & & & & & & & \\
\hline \multirow[t]{7}{*}{ 13. August } & Brot & 440 & 351,1 & 7,84 & 49,05 & 6,94 & 292,03 & 5,04 & \\
\hline & id. & 82 & 65,5 & 1,46 & 9,14 & 1,29 & 54,05 & 0,93 & 1125 \\
\hline & & 305 & 243,4 & 5,44 & 34 & 4,81 & 201,04 & 3,49 & - \\
\hline & Thunfisch & 47 & 24,8 & 2,03 & 12,06 & 11,43 & 0,04 & 0,54 & - \\
\hline & & $\begin{array}{r}57 \\
1990\end{array}$ & $\begin{array}{r}30 \\
351\end{array}$ & $\begin{array}{r}2,46 \\
2035\end{array}$ & 15,3 & 13,86 & $\begin{array}{r}0,05 \\
\end{array}$ & $\begin{array}{r}0,65 \\
\end{array}$ & $\begin{array}{r}1625 \\
750\end{array}$ \\
\hline & Suppe & 1220 & 351 & 10,35 & 64,88 & 50,32 & 213,44 & 17,32 & 750 \\
\hline & Summe & 2151 & 1065,8 & 29,61 & 184,97 & 88,65 & 760,65 & 27,97 & 3500 \\
\hline \multirow[t]{6}{*}{ 14. August } & Brot & 464 & 370,3 & 8,25 & 51,62 & 7,32 & 305,85 & 5,31 & - \\
\hline & id. & 116 & 32,6 & 2,06 & 12,93 & 1,83 & 76,46 & 1,32 & 1750 \\
\hline & & 265 & 211,5 & 4,72 & 29,54 & 4,89 & 174,68 & 3,03 & 500 \\
\hline & Melone & 976,5 & 13,3 & 0,38 & 2,39 & 3,50 & 5,61 & 1,73 & - \\
\hline & $\begin{array}{l}\text { Suppe } \\
\text { Käse }\end{array}$ & $\begin{array}{r}932 \\
36\end{array}$ & $\begin{array}{c}192,4 \\
24\end{array}$ & 5,52 & $\begin{array}{r}34,56 \\
9,69\end{array}$ & 19,03 & 125,92 & $\begin{array}{r}12,82 \\
1,49\end{array}$ & - \\
\hline & Summe & 27895 & 9041 & 2974 & 14073 & 4789 & 69008 & 9570 & 9950 \\
\hline & & & & & & & & & \\
\hline \multirow[t]{6}{*}{ 15. August } & Brot & 188 & 150,1 & 3,35 & 20,95 & 2,96 & 123,92 & 2,15 & - \\
\hline & id. & 136 & 108,6 & 2,42 & 15,16 & 2,14 & 89,64 & 1,55 & $\cdot 375$ \\
\hline & id. & 292 & 233 & 5,20 & 32,55 & 4,01 & 192,47 & 3,34 & - \\
\hline & Suppe & 1060 & 217,9 & 7,92 & 49,54 & 33,9 & 172,68 & 19,97 & 1625 \\
\hline & Käse & 47 & 731,3 & 2,37 & 12,05 & 14,69 & 2,03 & 1,95 & 750 \\
\hline & Summe & 1723 & 740,9 & 21,26 & 130,85 & 58,30 & 580,74 & 28,96 & 2750 \\
\hline
\end{tabular}

durch Extraction mit Aether gewonnenen 312,6 auch stickstoffhaltige Substanzen einbegriffen sein müssen, haben wir andere Stickstoffbestimmungen am entfetteten Käse angestellt und die resultirende Zahl als Albuminoide eingerechnet. In 312,6 mit Aether extrahirten Fetten findet man stickstoffhaltige Substanzen, welche 7,45 Stickstoff enthalten. 
Tabelle XIII. Nahrung des Weibes.

\begin{tabular}{|c|c|c|c|c|c|c|c|c|c|}
\hline \multirow{2}{*}{ Datum } & \multicolumn{2}{|c|}{ Speisen } & \multirow{2}{*}{ 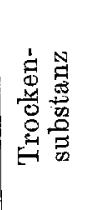 } & \multirow{2}{*}{ 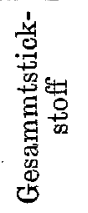 } & \multirow{2}{*}{ 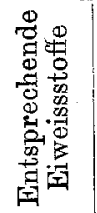 } & \multirow{2}{*}{$\underset{\stackrel{D}{ \pm}}{\stackrel{D}{0}}$} & \multirow{2}{*}{ 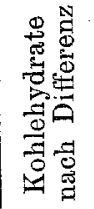 } & \multirow{2}{*}{ 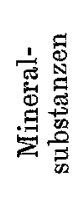 } & \multirow{2}{*}{ 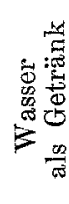 } \\
\hline & Qualität & $\begin{array}{l}\text { Quan- } \\
\text { tität }\end{array}$ & & & & & & & \\
\hline \multirow{8}{*}{ 13. August } & Brot & 310 & 247,4 & 5,52 & 34,5 & 4,89 & 204,34 & 3,55 & \\
\hline & id. & 73,5 & 58,7 & 1,29 & 3,1 & 1,16 & 48,44 & 0,84 & 625 \\
\hline & id. & 64 & 51 & 1,13 & 7,1 & 1,01 & 42,18 & 0,73 & \\
\hline & & 250 & 199,5 & 4,44 & 27,8 & 3,24 & 164,79 & 2,86 & 1000 \\
\hline & Thunfisch & 43 & 22,7 & 1,85 & 11,6 & 10,46 & 0,04 & 0,49 & 375 \\
\hline & & 35 & 18,5 & 1,51 & 9,4 & 8,51 & 0,03 & 0,40 & - \\
\hline & Suppe & 900 & 259 & 7,65 & 47,86 & 37,12 & 161,14 & 12,79 & \\
\hline & Summe & 1675,5 & 856,8 & 23,39 & 146,36 & 87,09 & 620,96 & 21,60 & 2000 \\
\hline \multirow[t]{7}{*}{ 14. August } & Brot & 282 & 225,1 & 5,02 & 31,4 & 4,45 & 185,88 & 3,23 & \\
\hline & id. & 73 & 58,3 & 1,29 & 8,1 & 1,15 & 48,11 & 0,83 & \\
\hline & id. & 221 & 176,6 & 4,06 & 25,4 & 3,48 & 145,97 & 2,53 & 500 \\
\hline & Melone & 810,5 & 11 & 0,31 & 1,98 & 2,90 & 4,63 & 1,41 & 500 \\
\hline & Suppe & 910 & 187,9 & 5,39 & 33,69 & 18,60 & 122,95 & 12,52 & 375 \\
\hline & Käse & 30 & 19,5 & 1,51 & 8,07 & 9,37 & 1,30 & 1,24 & \\
\hline & Summe & $2326,5 \mid$ & 678,4 & 17,58 & 108,64 & 39,95 & 508,54 & 21,76 & 1375 \\
\hline \multirow[t]{6}{*}{ 15. August } & Brot & 113,5 & 90,6 & 2,01 & 12,6 & 1,79 & 74,81 & 1,30 & \\
\hline & id. & 89,5 & 71,5 & 1,58 & 9,9 & 1,41 & 58,99 & 1,02 & \\
\hline & id. & $225^{\circ}$ & 179,6 & 4,01 & 25,1 & 3,55 & 148,31 & 2,57 & 250 \\
\hline & Suppe & 1018 & 209,2 & 7,61 & 47,58 & 32,56 & 109,87 & 19,17 & 750 \\
\hline & Käse & 37 & 24,6 & 1,86 & 9,96 & 11,56 & 1,60 & 1,53 & \\
\hline & Summe & 1483 & 575,5 & 17,07 & 105,14 & 50,87 & 393,58 & 25,59 & 1500 \\
\hline
\end{tabular}

Tabelle XIV. Ernährung des Knaben.

\begin{tabular}{|c|c|c|c|c|c|c|c|c|c|}
\hline \multirow{2}{*}{ Datum } & \multicolumn{2}{|c|}{ Speisen } & \multirow{2}{*}{ 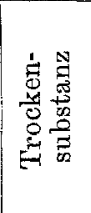 } & \multirow{2}{*}{ 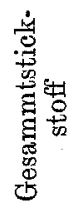 } & \multirow{2}{*}{ 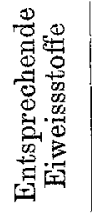 } & \multirow{2}{*}{ 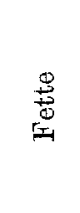 } & \multirow{2}{*}{ 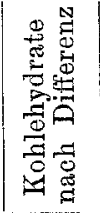 } & \multirow{2}{*}{ 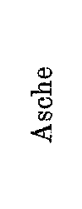 } & \multirow{2}{*}{ 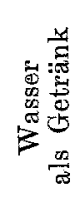 } \\
\hline & Qualität & $\begin{array}{l}\text { Quan- } \\
\text { tität }\end{array}$ & & & & & & & \\
\hline \multirow{7}{*}{ 13. August } & Brot & 195 & 155,6 & 3,47 & 21,7 & 3,07 & 128,53 & 2,23 & - \\
\hline & id. & 76 & 60,7 & 1,35 & 8,4 & 1,20 & 50,09 & 0,87 & 875 \\
\hline & & 140 & 111,8 & 2,49 & 15,6 & 2,21 & 92,28 & 1,60 & - \\
\hline & Thunfisch & 40 & 21,1 & 1,72 & 10,8 & 9,73 & 0,04 & 0,46 & $=$ \\
\hline & & 42 & 22,1 & 1,80 & 11,3 & 10,21 & 0,04 & 0,48 & 625 \\
\hline & Suppe & 726 & 208,9 & 6,17 & 38,61 & 29,95 & $130^{\circ}$ & 10,32 & - \\
\hline & Summe & 1219 & 580,02 & 16,99 & 106,41 & 56,37 & 400,98 & 15,96 & 1500 \\
\hline
\end{tabular}


Ueber die Nahrungs- und Stoffwechselbilanz des italienischen Bauers. 235

\begin{tabular}{|c|c|c|c|c|c|c|c|c|c|}
\hline \multirow{2}{*}{ Datum } & \multicolumn{2}{|c|}{ Speisen } & \multirow{2}{*}{ 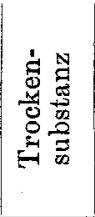 } & \multirow{2}{*}{ 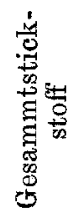 } & \multirow{2}{*}{ 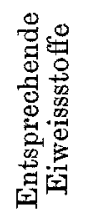 } & \multirow{2}{*}{ 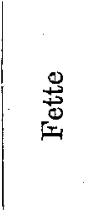 } & \multirow{2}{*}{ 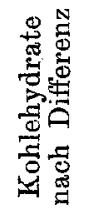 } & \multirow{2}{*}{ 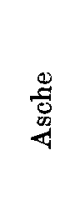 } & \multirow{2}{*}{ 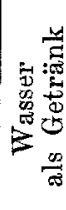 } \\
\hline & Qualität & $\begin{array}{l}\text { Quan- } \\
\text { tität }\end{array}$ & & & & & & & \\
\hline \multirow[t]{7}{*}{ 14. August| } & Brot & 233 & 186 & 4,15 & 25,9 & 3,67 & 153,58 & 2,67 & - \\
\hline & id. & 78 & 62,3 & 1,39 & 8,7 & 1,23 & 51,41 & 0,89 & 875 \\
\hline & & 36 & 28,8 & 0,64 & 4 & 0,56 & 23,73 & 0,41 & - \\
\hline & Melone & 746 & 10,2 & 0,29 & 1,82 & 2,67 & 4,26 & 1,30 & 125 \\
\hline & Suppe & $\begin{array}{l}445 \\
222\end{array}$ & $\begin{array}{l}92 \\
47,5\end{array}$ & 2,64 & $\begin{array}{r}16,5 \\
8,1\end{array}$ & $\begin{array}{l}9,10 \\
4,53\end{array}$ & $\begin{array}{l}60,12 \\
30\end{array}$ & 6,12 & - \\
\hline & Käse & 38 & 25,3 & 2,99 & 10,2 & 11,87 & 1,64 & 1,58 & - \\
\hline & Summe & 1798 & 452,1 & 13,39 & $75,22^{\circ}$ & 33,63 & 324,74 & 16,02 & 1000 \\
\hline \multirow[t]{6}{*}{ 15. August } & Brot & 98 & 78,2 & 1,74 & 10,9 & 1,54 & 64,59 & 1,12 & - \\
\hline & id. & 69,5 & 55,5 & 1,23 & 7,7 & 1,09 & 45,81 & 0,79 & - \\
\hline & id. & 150 & 119,7 & 2,67 & 16,7 & 2,36 & 98,87 & 1,71 & 125 \\
\hline & $\begin{array}{l}\text { Suppe } \\
\text { Käse }\end{array}$ & 457 & 93,9 & 3,41 & 21,36 & 14,6 & 49,32 & 8,60 & 375 \\
\hline & Käse & 37 & 24,6 & 2,92 & 9,96 & 11,56 & 1,60 & 1,53 & 375 \\
\hline & Summe & 811,5 & 371,9 & 11,99 & 66,62 & 31,15 & 260,19 & 13,75 & 875 \\
\hline
\end{tabular}

8) Die Ausscheidung dureh den Koth ist für alle drei Versuchspersonen in der Tabelle $\mathrm{XV}$ zusammengestellt.

Tabelle XV. Elimination mit dem Kothe.

\begin{tabular}{|c|c|c|c|c|c|c|c|c|}
\hline Datum & 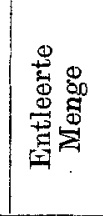 & 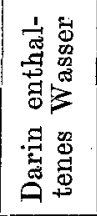 & 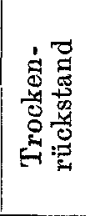 & 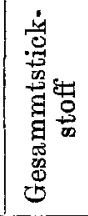 & 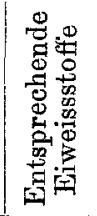 & 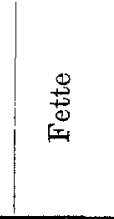 & 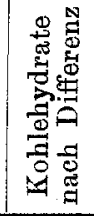 & $\underset{\mathscr{D}}{\tilde{D}}$ \\
\hline 13. August $\left\{\begin{array}{l}\text { Mann } \\
\text { Weib } \\
\text { Knabe }\end{array}\right.$ & $\begin{array}{l}322,4 \\
393,5 \\
123,76\end{array}$ & $\begin{array}{r}283,42 \\
341,73 \\
86,59\end{array}$ & $\begin{array}{l}38,98 \\
51,77 \\
37,17\end{array}$ & $\begin{array}{l}2,306 \\
2,65 \\
0,861\end{array}$ & $\begin{array}{r}14,41 \\
16,56 \\
5,38\end{array}$ & $\begin{array}{l}6,21 \\
9,21 \\
3,767\end{array}$ & $\begin{array}{r}13,83 \\
18,84 \\
3,87\end{array}$ & $\begin{array}{l}4,53 \\
7,16 \\
4,15\end{array}$ \\
\hline 14. August $\left\{\begin{array}{l}\text { Mann } \\
\text { Weib } \\
\text { Kuabe }\end{array}\right.$ & $\begin{array}{l}211,5 \\
372,4 \\
157\end{array}$ & $\begin{array}{l}185,72 \\
320,07 \\
130,48\end{array}$ & $\begin{array}{l}25,78 \\
52,33 \\
26,52\end{array}$ & $\begin{array}{l}1,51 \\
2,925 \\
1,61\end{array}$ & $\begin{array}{r}9,43 \\
18,28 \\
10,06\end{array}$ & $\begin{array}{r}6,552 \\
11,909 \\
4,455\end{array}$ & $\begin{array}{r}5,90 \\
13,19 \\
8,47\end{array}$ & $\begin{array}{l}3,90 \\
8,95 \\
3,53\end{array}$ \\
\hline 15. August $\left\{\begin{array}{l}\text { Mann } \\
\text { Weib } \\
\text { Knabe }\end{array}\right.$ & $\begin{array}{l}203,2 \\
218 \\
371,5\end{array}$ & $\begin{array}{l}127,58 \\
190,64 \\
323,93\end{array}$ & $\begin{array}{l}76,62 \\
27,36 \\
47,57\end{array}$ & $\begin{array}{l}2,60 \\
1,344 \\
2,746\end{array}$ & $\begin{array}{r}16,25 \\
8,40 \\
17,16\end{array}$ & $\begin{array}{c}15,2111 \\
5,863 \\
9,811\end{array}$ & $\begin{array}{r}31,58 \\
8,80 \\
12,13\end{array}$ & $\begin{array}{r}12,57 \\
4,39 \\
8,46\end{array}$ \\
\hline
\end{tabular}


9) Fï r die durch den Harn stattgefundene A us scheidung legen wir die Tabelle XVI bei, die in derselben Weise wie Tabelle VI verfasst ist.

Tabelle XVI. Elimination mit dem Harne.

\begin{tabular}{|c|c|c|c|c|c|c|c|c|}
\hline Datum & 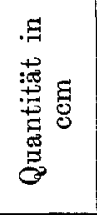 & 总 & 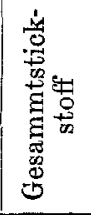 & 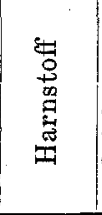 & 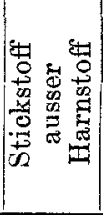 & 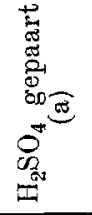 & 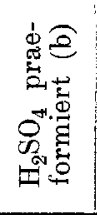 & 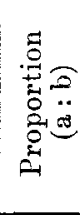 \\
\hline 13. August $\left\{\begin{array}{l}\text { Mann } \\
\text { Weib } \\
\text { Knabe }\end{array}\right.$ & $\begin{array}{l}1358,2 \\
1319 \\
588,6\end{array}$ & $\begin{array}{l}1026,7 \\
1022,5 \\
1026,7\end{array}$ & $\begin{array}{r}15,84 \\
12,59 \\
5,49\end{array}$ & $\begin{array}{l}33,50 \\
25 \\
9,455\end{array}$ & $\begin{array}{l}0,16 \\
0,89 \\
1,07\end{array}$ & $\begin{array}{l}3,9569 \\
2,7152 \\
1,3183\end{array}$ & $\begin{array}{l}0,3778 \\
0,2202 \\
0,2112\end{array}$ & $\begin{array}{l}1: 10 \\
1: 12 \\
1: 6\end{array}$ \\
\hline 14. August $\left\{\begin{array}{l}\text { Mann } \\
\text { Weib } \\
\text { Knabe }\end{array}\right.$ & $\begin{array}{c}1180 \\
1454 \\
676,4\end{array}$ & $\begin{array}{l}1031,2 \\
1017,2 \\
1022,2\end{array}$ & $\begin{array}{r}16,90 \\
9,72 \\
7,27\end{array}$ & $\begin{array}{l}34,61 \\
20,10 \\
12,11\end{array}$ & $\begin{array}{l}0,70 \\
0,30 \\
1,61\end{array}$ & $\begin{array}{l}4,0938 \\
2,2269 \\
1,3819\end{array}$ & $\begin{array}{l}0,1852 \\
0,1721 \\
0,1168\end{array}$ & $\begin{array}{l}1: 22 \\
1: 12 \\
1: 11\end{array}$ \\
\hline 15. August $\left\{\begin{array}{l}\text { Mann } \\
\text { Weib } \\
\text { Knabe }\end{array}\right.$ & $\begin{array}{l}1181.6 \\
1460 \\
630,2\end{array}$ & $\begin{array}{l}1029,5 \\
1019 \\
1026\end{array}$ & $\begin{array}{r}18,30 \\
12,64 \\
6,98\end{array}$ & $\begin{array}{c}35,56 \\
24,69 \\
-\end{array}$ & $\begin{array}{l}1,66 \\
1,09 \\
-\end{array}$ & $\begin{array}{l}3,9952 \\
2,5847 \\
1,4037\end{array}$ & $\begin{array}{l}0,2760 \\
0,2449 \\
0,1680\end{array}$ & $\begin{array}{l}1: 14 \\
1: 10 \\
1: 8\end{array}$ \\
\hline
\end{tabular}

10) B i l a n z. Tabelle XVII für den Stickstoff.

Tabelle XVIII für die Kohlenhydrate.

Tabelle XIX für Wasser.

Tabelle XVII. Stickstoffbilanz.

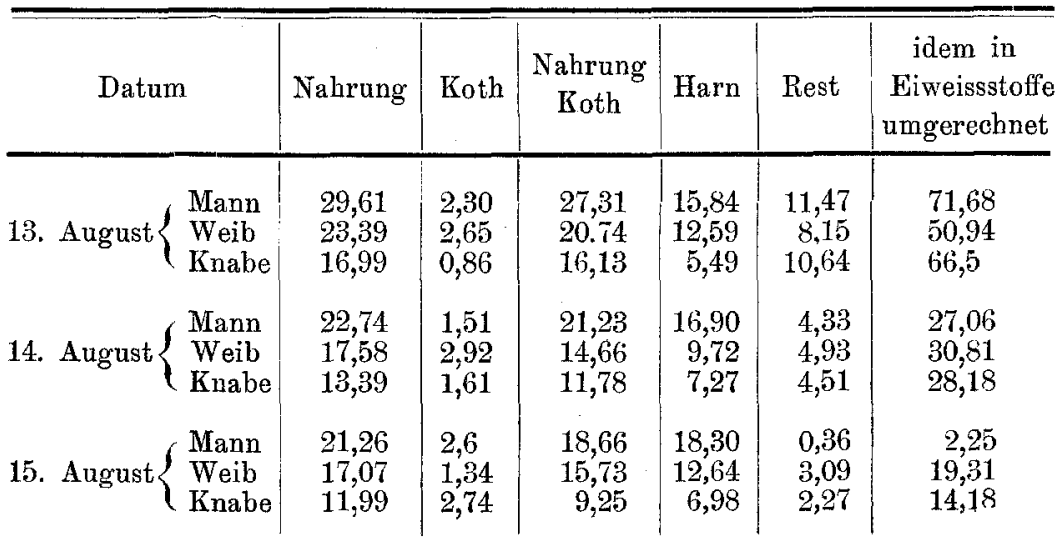


Ueber die Nahrungs- und Stoffwechselbilanz des italienischen Bauers. 237

Tabelle XVIII.

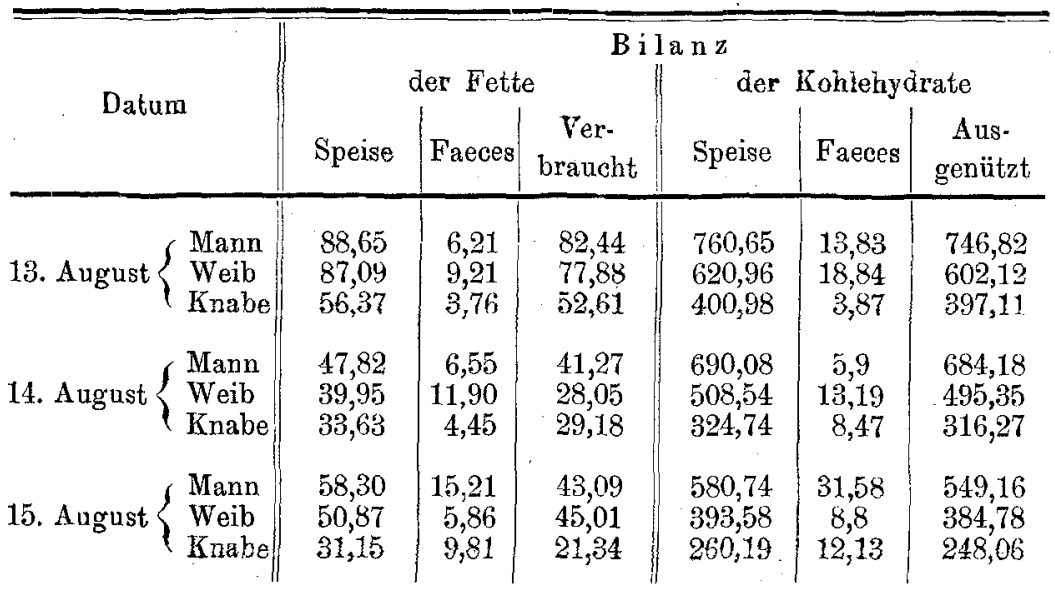

Tabelle XIX. Wasserbilanz.

\begin{tabular}{|c|c|c|c|c|c|c|c|}
\hline Datura & 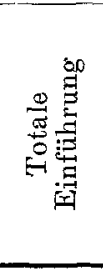 & 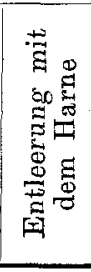 & 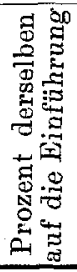 & 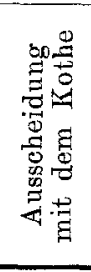 & 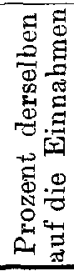 & 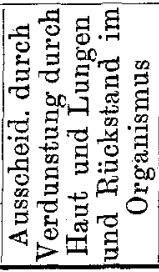 & 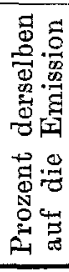 \\
\hline Mann & 4585,2 & 1358,2 & 29 & 283,42 & 7 & 2943.58 & 64 \\
\hline 13. August Weib & 2818,6 & 1319 & 46 & 341,73 & 13 & 1158,27 & 41 \\
\hline ( Knabe & 2138,8 & 588,6 & 27 & 86,59 & 5 & $14.63,61$ & 68 \\
\hline ( Mann & 4135,4 & 1180 & 28 & 185,72 & 6 & 2769,68 & 66 \\
\hline 14. August $\{$ Weib & 3023,1 & 1454 & 48 & 320,07 & 11 & 1249,03 & 41 \\
\hline IKnabe & 2345,9 & 676,4 & 28 & 130,48 & 22 & 1539,02 & 50 \\
\hline Mann & 3732,1 & 1181,6 & 31 & 127,58 & 4 & 2422,92 & 65 \\
\hline 1o. August $\{$ Weib & 2407,5 & $1460^{\circ}$ & 60 & 190,64 & 8 & 756,9 & 31 \\
\hline Knabe & 1314,6 & 630,2 & 47 & 323,93 & 26 & 360,47 & 27 \\
\hline
\end{tabular}

11) In der Tabelle $X X$ führen wir den Prozentge halt an Fett, Stickstoff, und Kohlenhydratean, welche im Kothe wiedererschienen sind. 
Tabelle XX. Prozent von Stickstoff, Fett und Kohlebydrate, welche in dem Kothe gefunden werden.

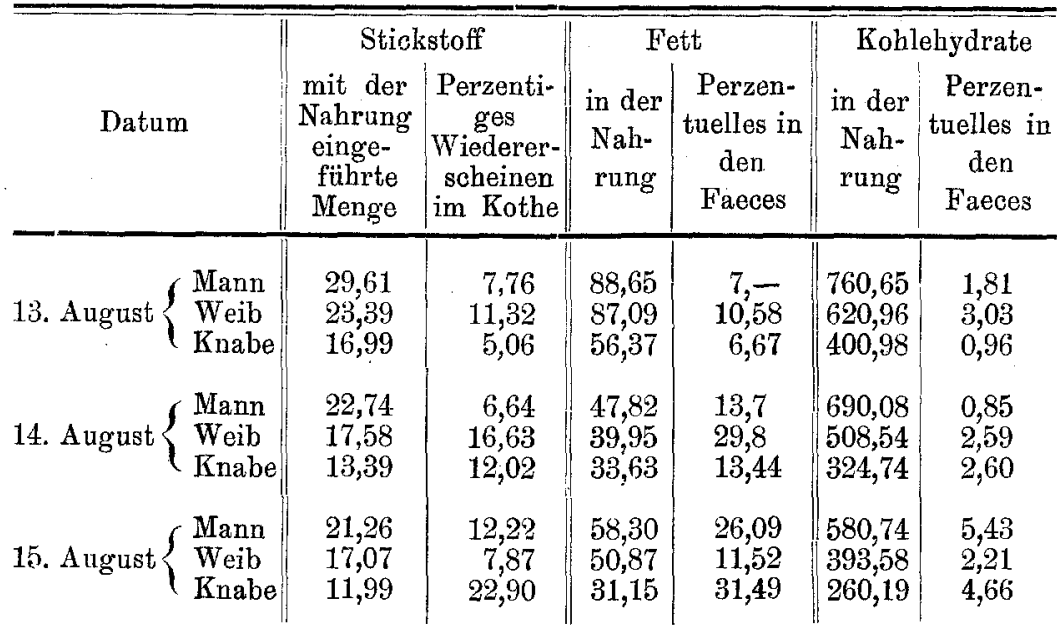

\section{\$ IV. Vergleich zwischen Winter- und Sommerbilanz.}

Ein Blick auf die zwei folgenden Tabellen geniigt, um eine Vorstellung zu erwecken von der Quantität eingeführter und assimilirter Nahrungsmittel und von der Zahl Calorien, welche sich ans den Speisen in den zwei Zeitperioden entwickelten, während welcher unsere Versuche stattfanden.

Tabelle XXI.

\begin{tabular}{|c|c|c|c|c|c|c|c|}
\hline & Datum & 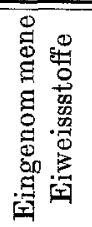 & 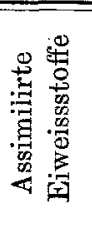 & 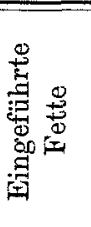 & 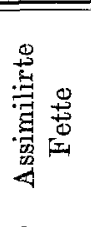 & 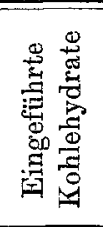 & 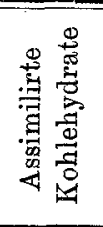 \\
\hline Mann & $\left\{\begin{array}{cc}\text { März } & 3 . \\
7 & 4 . \\
& 5 .\end{array}\right.$ & $\begin{array}{l}79,25 \\
80,54 \\
88,94\end{array}$ & $\begin{array}{l}49,49 \\
61,49 \\
78,54\end{array}$ & $\begin{array}{l}74,05 \\
54,75 \\
63,19\end{array}$ & $\begin{array}{l}64,40 \\
53,32 \\
56,36\end{array}$ & $\begin{array}{l}537,73 \\
648,28 \\
551,44\end{array}$ & $\begin{array}{l}479,98 \\
614,68 \\
513,68\end{array}$ \\
\hline Weib & $\left\{\begin{array}{cc}\text { März } & 3 . \\
" & 4 . \\
" & 5 .\end{array}\right.$ & $\begin{array}{l}60,35 \\
74,93 \\
75,19\end{array}$ & $\begin{array}{l}46,32 \\
60,92 \\
56,06^{2}\end{array}$ & $\begin{array}{l}48,60 \\
51,23 \\
44,17\end{array}$ & $\begin{array}{l}42,05 \\
50,23 \\
35,98\end{array}$ & $\begin{array}{l}392,11 \\
589,68 \\
487,33\end{array}$ & $\begin{array}{l}370,24 \\
557,82 \\
456,63\end{array}$ \\
\hline Knabe & $\left\{\begin{array}{cc}\text { März } & 3 . \\
\Rightarrow & 4 . \\
\Rightarrow & 5 .\end{array}\right.$ & $\begin{array}{l}38,45 \\
48,11 \\
64,97\end{array}$ & $\begin{array}{l}35,37 \\
40,96 \\
59,74\end{array}$ & $\begin{array}{l}36,95 \\
33,66 \\
42,05\end{array}$ & $\begin{array}{l}31,78 \\
31,19 \\
34,38\end{array}$ & $\begin{array}{l}218,49 \\
386,57 \\
400,89\end{array}$ & $\begin{array}{l}191,14 \\
373,98 \\
398,72\end{array}$ \\
\hline
\end{tabular}


Ueber die Nahrungs- und Stoffwechselbilanz des italienischen Bauers. 239

\begin{tabular}{|c|c|c|c|c|c|c|c|}
\hline & Datum & 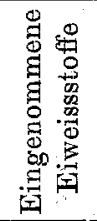 & 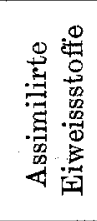 & 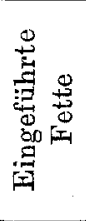 & 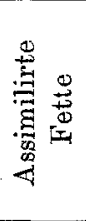 & 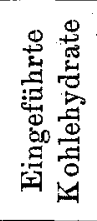 & 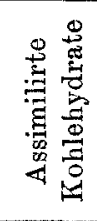 \\
\hline Mann & $\left\{\begin{array}{cc}\text { Angust } 13 . \\
" \quad 14 . \\
" \quad 15 .\end{array}\right.$ & $\begin{array}{l}184,97 \\
140,73 \\
130,85\end{array}$ & $\begin{array}{l}170,56 \\
131,30 \\
114,60\end{array}$ & $\begin{array}{l}88,65 \\
47,82 \\
58,30\end{array}$ & $\begin{array}{l}82,44 \\
41,27 \\
43,09\end{array}$ & $\begin{array}{l}760,65 \\
690,08 \\
580,74\end{array}$ & $\begin{array}{l}746,82 \\
684,18 \\
549,16\end{array}$ \\
\hline Weib & $\left\{\begin{array}{cc}\text { August } 13 . \\
n & 14 . \\
" & 15 .\end{array}\right.$ & $\begin{array}{l}146,36 \\
108,64 \\
105,14\end{array}$ & $\begin{array}{r}129,80 \\
90,36 \\
96,72\end{array}$ & $\begin{array}{l}87,09 \\
39,95 \\
50,87\end{array}$ & $\begin{array}{l}77,88 \\
28,05 \\
45,01\end{array}$ & $\begin{array}{l}620,96 \\
508,54 \\
393,58\end{array}$ & $\begin{array}{l}602,12 \\
495,35 \\
384,78\end{array}$ \\
\hline Knabe & $\left\{\begin{array}{cc}\text { August } 13 . \\
" & 14 . \\
" & 15 .\end{array}\right.$ & $\begin{array}{r}106,41 \\
75,22 \\
66,62\end{array}$ & $\begin{array}{r}101,04 \\
65,16 \\
49,46\end{array}$ & $\begin{array}{l}56,37 \\
33,63 \\
31,15\end{array}$ & $\begin{array}{l}52,61 \\
29,18 \\
21,34\end{array}$ & $\begin{array}{l}400,98 \\
324,74 \\
260,19\end{array}$ & $\begin{array}{l}397,11 \\
316,27 \\
248,06\end{array}$ \\
\hline
\end{tabular}

(Die Tabelle XXII steht auf der nächsten Seite.)

Bemerkenswerth ist, dass im Winter die tägliche durchschnitt. liche Ausgabe für die ganze Familie Fes. 1,00, im Sommer dagegen Fcs. 1,44 beträgt. Der Unterschied in den Nährstoffen giebt sich kund hauptsächlich in den Eiweissstoffen, welche im Verbältnisse zum Brote und zur Suppe fast um das Doppelte vermehrt sind. Das auffälligste Ergebniss unserer Versuche liegt in dem grossen Unterschiede zwischen der Sommer und Winterbilanze. Im Winter begegnen wir einem beträchtlichen Deficit an assimilirtem Stickstoffe mit Rücksicht auf den Verlust mit dem Harne, mit anderen Worten die Eiweissstoffe werden mit der Nahrung in ungenügender Menge eingeführt. Dagegen finden wir im Sommer entschieden Sparung von Eiweissstoffen und so gleicht der Organismus im Sommer die erlittenen Verluste wieder aus. Bedenkt man, dass bekanntlich im Winter der Appetit sich lebhafter geltend macht und dass die Verdauungskraft eine regere ist, so liegt hier offenbar nicht eine physiologische Erscheinung vor, sondern zweifellos eine Umkehrung physiologischer Zustände, die durch die ökonomischen Verhältnisse herbeigefuhrt ist.

Hirsehfeld, K u magawa und andere behaupten, dass die von Vo.it angenommene tägliche Ration von 118 Gramm Eiweissstoffen nicht nothwendig ist, dass ein Mann eine Zeit lang sich im Stickstoffgleichgewicht auch mit 40-50 Gr. Eiweiss erhalten kann, vorausgesetzt, dass er gleichzeitig eine beträchtliche Mepge Fette und Kohlenhydrate zu sich nimmt, während anderer- 


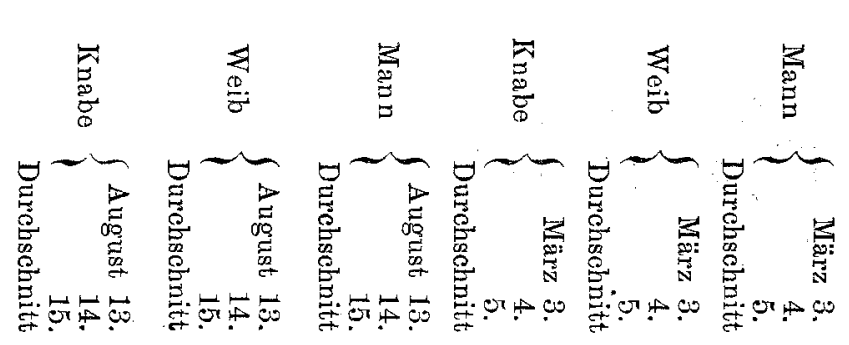

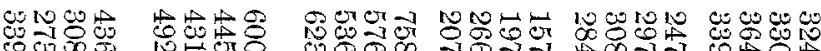
at

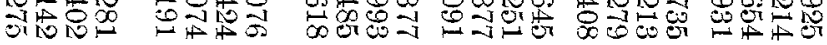

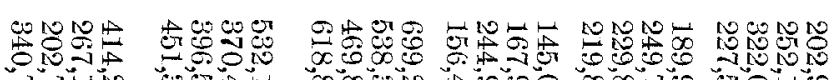

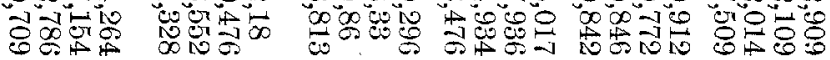

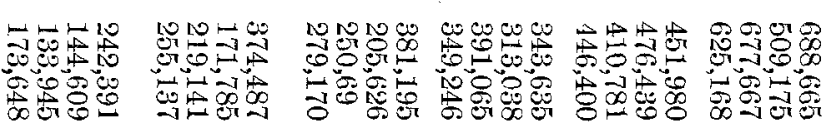

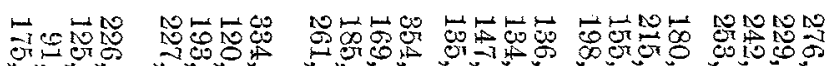

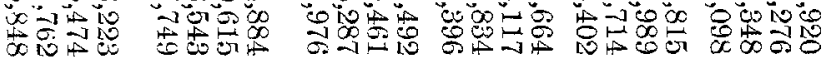

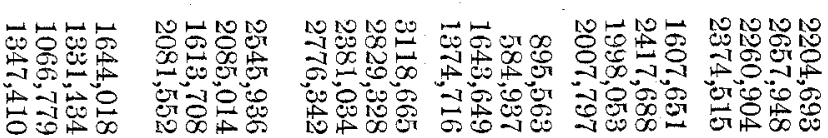

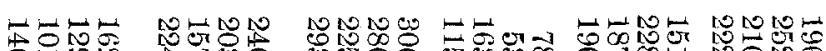
ज.

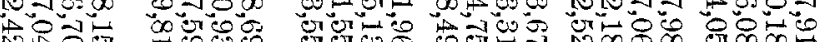

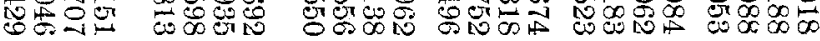

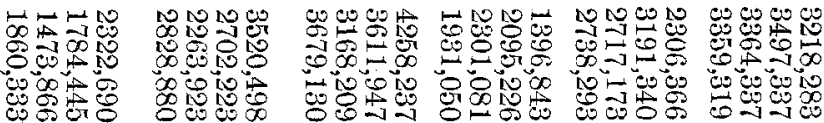

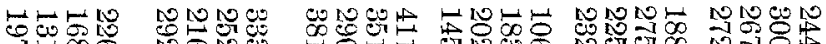

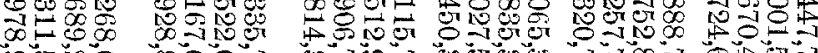

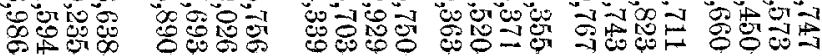


seits auch mit einer ausreichenden Zufuhr von Eiweisssubstanz $(78,112$ Gramm) und mit der von V o it versehriebenen Quantität anderer Nahrungsmittel ein beträchtlicher Verlust an Körpereiweiss eintrat, wenn die Nahrung für die Caloriebedürfnisse des Organismus nicht genügte. M u $\mathrm{k}$ hat versetzt, dass eine solche eiweissarme Nahrung nur für kurze Zeit ansreicht, dass sie bei längerer Fortsetzung eine Reibe von Störungen und Schäden im Organismus herbeiführt. H i r s ch f e l d erhebt den Einwand, dass dies für den Hund, an dem $\mathrm{M} \mathrm{u} \mathrm{n} \mathrm{k}$ experimentirt hat, und nicht für den Menschen zutrifft. Wir miissen diese Frage vom individuellen Standpunkte betrachten. Zweifellos giebt es einige Individuen, für welche die Behauptung von Hirschfeld, K u magawa und Briesacker sich bewabrheitet, aber für die Mehrzahl gilt $V o$ it's Norm. Ein individueller Fall von permanenter äusserster Einschränkung der Bilanze mit Erhaltung ausgezeichneter Gesundheit wurde in A lbertoni's Laboratorium von Buys (1) untersucht; der Stickstoff im Harne schwankte stets zwischen 6-7 Gramm. Buys fasst diesen Fall als eine Art Missbildung auf. Unserer Ansicht nach müssen gerade solche Fälle untersucht werden, aber man hüte sich, daraus Gesetze ableiten zu wollen.

Uebrigens haben diese Einwände keine Bedeutung für unsere Versuchspersonen, bei welehen das Stickstoffdeficit direct hervorgeht aus der Differenz zwischen dem assimilirten Stickstoffe und dem mit dem Harne verloren gegangenen. Der Mann verlor in den drei Märztagen 54,93 Gramm Eiweissstoffe und sparte davon 100,99 Gramm in den drei Augusttagen. Das Weib zeigte einen Eiweissverlust nur in zwei Tagen der Winterperiode, eine Ersparniss am dritten und ein erbebliches Ersparniss in der Sommerperiode; der Knabe erhielt sich im Bilanzgleichgewichte im Winter und sparte im Sommer. Der Stickstoffverlust mit dem Harne war beim Manne und Weibe grösser im Sommer entsprechend der erböhten Nahrungszufuhr; beim Manne ist die Durchschnittszahl in der Winterperiode 13,02, in der Sommerperiode 17,01; beim Weibe sind die entsprechenden Durchsehnittszahlen 9,32 und 11,65. Der Knabe zeigt geringfügige Schwankungen in der Stickstoffausscheidung

1) Ed. Buys, Un caso notevole di regime azotato scarso, abituale. Arch. It. de Biologie 1893 und Ann. di Chim. e di Farmacol. 1893 Bd. XVIII Seite 217. 
im Vergleiche zu Mann und Weib und eliminirt im Sommer weniger Stickstoff, wovon er eine beträchtliche Menge in sich aufspeichert. Die Menge aller eingeführten und assimilirten Nährstoffe schwankt an den verschiedenen Tagen der Sommer- und Winterperiode, ist aber immer erbeblicher im Sommer. Man findet keine beträchtlichen Unterschiede zwischen den Arbeits- und den Ruhetagen, wie aus den beigefügten Durchschnittstabellen zu ersehen ist.

Tabelle XXIII. Durchschnittszahlen der in beiden J a hreszeiten während der Arbeit und in der Rube eingefïhrten und assimilirten $N$ ä hrstoffe.

\begin{tabular}{l}
\hline L \\
\hline Mann
\end{tabular}

Bemerkenswerth ist die Thatsache, dass alle Nährstoffe während des Sommers constant in grösseren Mengen eingeführt und assimilirt wurden, wenn die Familie arbeitete als bei der Ruhe. Im Winter dagegen wurden die Eiweissstoffe immer spärlicher während der Arbeit eingeführt als bei der Rube; ein weiterer Beweis, dass eine Umkehrung der physiologischen Bilanz stattfindet.

Die Menge der Nahrung entsprechender Calorien, wie sie ans der Summe assimilirter Nahrungsmittel resultirt, ist immer hoch, gleich oder grösser als die Durchschnittszahl, grösser im Sommer als im Winter: 
Ueber die Nahrungs- und Stoffwechselbilanz des italienischen Bauers. 243

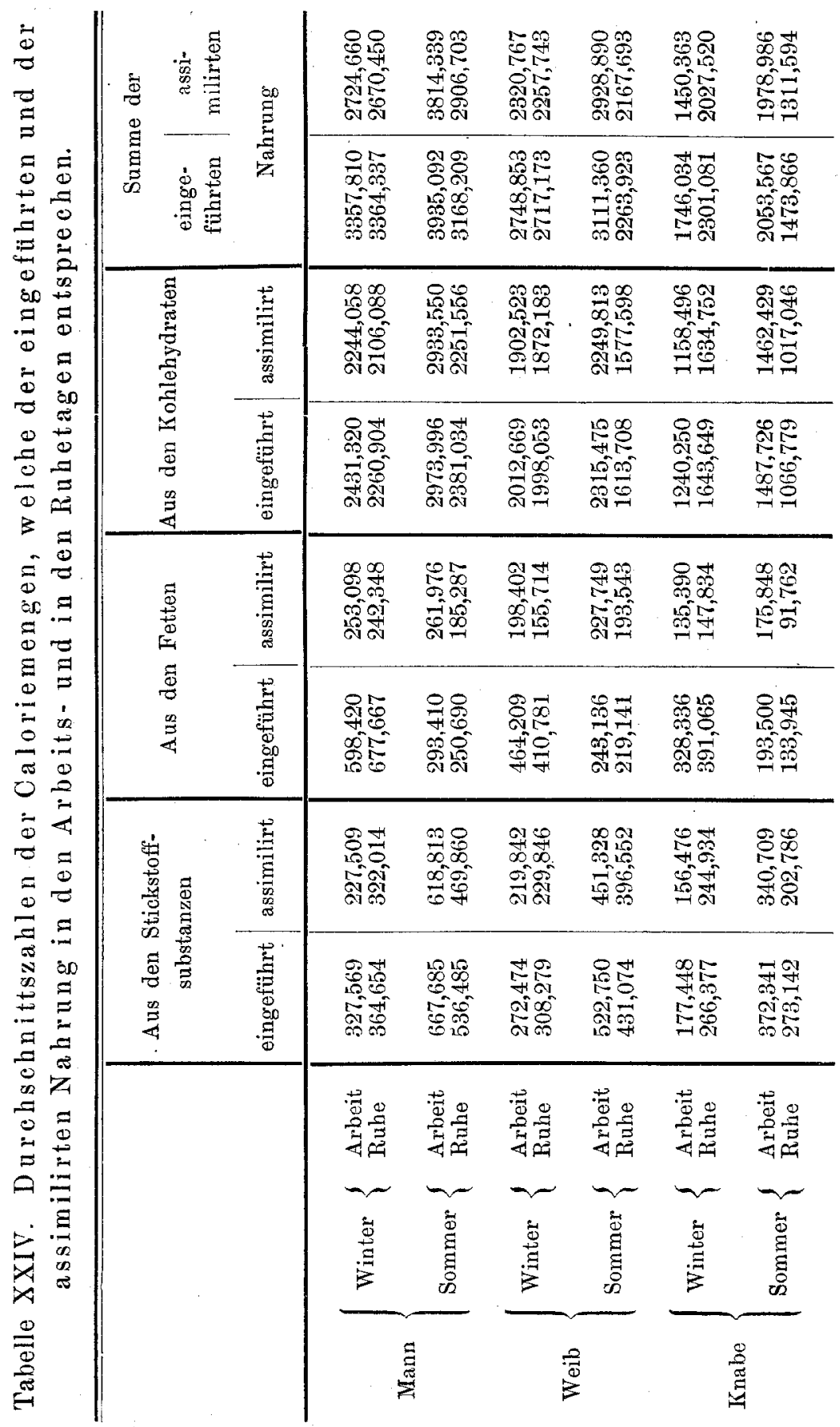


Bringt man die Summe der Calorien der assimilirten Nabrung in Beziehung zum Körpergewichte und zur Körperoberfläche, so ergiebt sich:

Tabelle XXV.

\begin{tabular}{|c|c|c|c|c|c|}
\hline & $\begin{array}{l}\text { Körper- } \\
\text { gewicht }\end{array}$ & $\begin{array}{c}\text { Körper- } \\
\text { oberfläche }\end{array}$ & $\begin{array}{c}\text { Calorien } \\
\text { pro Kilo im } \\
\text { Winter }\end{array}$ & $\begin{array}{c}\text { Calorien } \\
\text { pro Kilo im } \\
\text { Sommer }\end{array}$ & $\begin{array}{l}\text { Calorien } \\
\text { pro mq im } \\
\text { Winter } \\
\text { und Sommer }\end{array}$ \\
\hline Mann & 68,100 & $1,932,3$ & $\begin{array}{l}\text { Arbeit } \\
\text { Ruhe }\end{array}\left\{\begin{array}{l}40,0 \\
39,2\end{array}\right.$ & $\begin{array}{l}56,0 \\
46,2\end{array}$ & $\begin{array}{l}1410-1979 \\
1381-1504\end{array}$ \\
\hline Weib & 50,600 & $1,066,8$ & $\begin{array}{l}\text { Arbeit } \\
\text { Ruhe }\end{array}\left\{\begin{array}{l}45,8 \\
44,6\end{array}\right.$ & $\begin{array}{l}57,8 \\
42,8\end{array}$ & $\begin{array}{l}2175-2745 \\
2116-2081\end{array}$ \\
\hline Knabe & 34,800 & $0,504,6$ & $\begin{array}{l}\text { Arbeit } \\
\text { Ruhe }\end{array}\left\{\begin{array}{l}41,7 \\
58,2\end{array}\right.$ & $\begin{array}{l}56,8 \\
37,6\end{array}$ & $\begin{array}{l}2874-3919 \\
4018-2519\end{array}$ \\
\hline
\end{tabular}

Nur der Knabe bildet eine Ausnahme hinsichtlich der Zahl der Calorien, die von der im Winterrasttage assimilirten Nahrung repräsentirt sind. In allen anderen Fällen zeigt der Ruhetag eine niedrigere Zahl von der assimilirten Nahrung entsprechenden Calorien im Vergleiche zum Arbeitstage. Die Menge in absoluter und relativer Beziehung zur Körperoberfläche gebracht, ïbertrifft die von M a $\mathrm{fredi}$ an seinen Versuchspersonen erbaltenen Zahlen.

\section{$\S \mathbf{V}$.}

Eine zweite Untersuehungsreihe, die wir anstellten, betraf die Bestimmung der a usgeschiedenen Schwefel$\mathrm{s}$ ä ure, der Gesammtschwefelsäure und der mit den aromatischen Substanzen gepaarten, zu dem Zwecke die Intensität der Verwesungsprocesse im Darmkanale und die Quantität des vom Schwefel verbrannten Eiweiss, aus welchem fast die gesammte Schwefelsäure des Harnes stammt, festzustellen. Bekanntlich sehwankt die tägliche Ausscheidung der präformirten und der gepaarten Schwefelsäure von Person zu Person und steht in Beziehung zur Diät; die von den Autoren gemachten diesbeziigglichen Angaben spiegeln diese Einflüsse wieder und sind daher untereinander verschieden. Wir erinnern des Vergleiches halber, dass nach von den Velden bei gesunden Personen das Verhältniss zwischen 
Ueber die Nahrungs- und Stoffwechselbilanz des italienischen Bauers. 245

$1: 6,9$ und $1: 12,7$, durchschnittlich $1: 9,5$ schwankt; und die tägliche Ausscheidung der gepaarten Schwefelsäure beträgt 0,61-0,09.

$\mathrm{B}$ a u m an und $\mathrm{H}$ erter fanden erhebliche Schwankungen im normalen Verhältnisse zwischen $1: 4,2$ und $1: 27$, durchschnittlich $1: 15$.

G. Hop pe-Seyler fand bei gesunden Personen die normale tägliche Menge gepaarter Schwefelsäure zwisehen 0,175 und und 0,268 schwankend und jene der präformirten Schwefelsäure zwischen 2,085 und 3,426 , das Verhältniss $1: 11,4$ and $1: 12,4$, B i e r n a cki fand bei gewöhnlicher Diät von 95,3 gr. Eiweiss, 66,9 Fett, 477,7 Kohlenhydrate gr. 1,6393 Gesammtschwefelsäure, gr. 1,4975 präformirter, 0,1418 gepaarter, Verhältniss $1: 10,5$.

A lbertoni fand bei zwei an sich selbst bei gemischter vorwiegender Fleischkest angestellten Versuchen : gepaarte Schwefelsäure $0,1548-0,2300$, präformirte $3,117-4,5025$, totale $3,271-4,7325$, Verhältniss 1:23 und 1:19. Sein Schiuler Heinrich Pinzani fand an zwei Weibern bei gemischter Diät grr. 3,054 und 3,170 präformirte, gr. 0,27, 0,26 gepaarte Schwefelsäure, Verhältniss $1: 11$ and $1: 12$. Der Harn eines von $\mathrm{B} \mathrm{u} \mathrm{n} g$ e bei absoluter Fleischdiät angebaltenen jungen Mannes enthielt 4,674 gr Gesammtschwefelsäure und zwei Tage nach einer aus Brot, Butter and etwas Salz bestehenden Nahrung wurden nur 1,265 gr Gesammtschwefelsäure angetroffen.

Bei unseren Versuchspersonen war die Ausscheidung der präformirten Schwefelsäure beim Manne ïber $3 \mathrm{gr}$, böher im Sommer, und jene der gepaarten schwankte zwisehen 11-37 etgr pro die, das Verhältniss zwischen $1: 10$ und 11:26. Beim Weibe schwankte die präformirte Schwefelsäure um $2 \mathrm{gr}$ im Winter und 2,50 im Sommer, die gepaarte zwischen 11 und $28 \mathrm{ctgr}$, die Proportion zwischen $1: 6$ und $1: 19$.

Beim Knaben war die Ausscheidung der präformirten Schwefelsäure grösser im Winter als im Sommer und schwankte zwischen 1,31 und 1,99, die gepaarte zwischen 10 und 21 ctgr, das Verhältniss zwischen $1: 6$ und 1:16. Im Allgemeinen bewegten sich demnach die Bildung der aromatischen Substanzen und die Ausscheidung der Schwefelsäure in ihren verschiedenen Zuständen in normalen Grenzen. 
Albertoni u. Novi: Ueber die Nahrungs- und Stoffwechselbilanz etc.

\$ VI. Preis der Nahrung in den Winter- und Sommertagen.

\begin{tabular}{|c|c|}
\hline $\begin{array}{l}\text { Winterration für die ganze Familie } \\
\text { in } 3 \text { Tagen }\end{array}$ & $\begin{array}{c}\text { Sommerration für die ganze Familie } \\
\text { in } 3 \text { Tagen }\end{array}$ \\
\hline Maismehl Kilo 8 & Brot Kilo 5,055 \\
\hline Schweinefett gr 180 . & Thun gr 300 . . \\
\hline Bohnen gr $500 .$. & Käse $\mathrm{gr} 250$. . \\
\hline Mehiteig gr 500 . & Bohnen gr 500 . . \\
\hline Speck $\quad \mathrm{gr} 180$. & Mehlteig gr 1500 . . \\
\hline Häringe No. 2 . . & Schweinefett $\operatorname{gr} 200$. \\
\hline Kastanienmehl . & Eine Melone . . . \\
\hline Salz gr 250 . & Salz gr 250 . \\
\hline Fes. 3,00 & Fes. 4,39 \\
\hline Eiweissstoffe $\mathrm{gr} \quad 610,73$ & Eiweissstoffe \\
\hline Fette $\quad " 448,65$ & Fett $\quad, \quad 493,83$ \\
\hline Kohlehydrate , 4212,46 & Kohlehydrate $, 4540,46$ \\
\hline
\end{tabular}

Wenn man nun durch die Daten, welche wir gesammelt haben, eine Bilanz der von uns als Objekt unserer Studien genommenen Familie herstellen will, nach der Beobachtung, dass sie einen jährlichen Ertrag von Fr. 486,40 geben, kann man die Ausgaben wie folgend sammeln :

I0-Kost, Wintertypus von September

bis April, 242 Tage (1 Fr. pro Tag) Fr. 242,-

Sommertypus Mai-August, 123 Tage

(Fr. 1,44 pro Tag) Fr. 177,12.

Minus 16 Tage schlechterer Kost

obne Suppe und Zukost

d. h. ohne $\left\{\begin{array}{l}\text { Käse . Fr. } 0,166 \\ \text { Thun . . , 0, } 20 \\ \text { Schweinsfett "0,134 } \\ \text { Mehlteig . "0, } 0,25 \\ \text { Bohnen. . } 0,133 \\ \text { Fr. } 0,883\end{array}\right.$

Also Fr. $177,12-$ Fr. $0,883 \times 16=$ Fr. $163,-$

II. Wohnung: zwei Zimmer

III. Kleidung a. s. w.

Fr. $405,-$

$" 60,-$

\begin{tabular}{cr} 
Fr. $\quad 21,40$ \\
\hline Fr. 486,40
\end{tabular} 